\title{
VARIABLE STARS AND STELLAR POPULATIONS IN ANDROMEDA XXI: II. ANOTHER MERGED GALAXY SATELLITE OF M31?*
}

\author{
FELICE CUSANO ${ }^{1}$, ALESSIA GAROFALO ${ }^{1,2}$, GISELLA CLEMENTINI ${ }^{1}$, MICHELE \\ CIGNONI $^{3}$, LUCIANA FEDERICI ${ }^{1}$, MARCELLA MARCONI ${ }^{4}$, ILARIA MUSELLA ${ }^{4}$, \\ VINCENZO RIPEPI ${ }^{4}$, ROBERTO SPEZIALI $^{5}$, ELEONORA SANI ${ }^{6,7}$, ROBERTO $^{2}$ \\ MERIGHI ${ }^{1}$ \\ ${ }^{1}$ INAF- Osservatorio Astronomico di Bologna, Via Ranzani 1, I - 40127 Bologna, Italy \\ felice.cusano@oabo.inaf.it \\ 2 The Observatories of the Carnegie Institution of Washington, 813 Santa Barbara Street, Pasadena, CA \\ 91101, USA \\ ${ }^{3}$ Space Telescope Science Institute, 3700 San Martin Drive, Baltimore, MD 21218, USA \\ ${ }^{4}$ INAF- Osservatorio Astronomico di Capodimonte, Salita Moiariello 16, I- 80131 Napoli, Italy \\ ${ }^{5}$ INAF- Osservatorio Astronomico di Roma, Via di Frascati 3300040 Monte Porzio Catone, Italy \\ ${ }^{6}$ INAF - Osservatorio Astronomico di Arcetri, Largo Enrico Fermi 5, I - 50125 Firenze \\ ${ }^{7}$ ESO, Alonso de Cordova 3107, Casilla 19001, Vitacura, Santiago 19, Chile
}

\begin{abstract}
$B$ and $V$ time-series photometry of the M31 dwarf spheroidal satellite Andromeda XXI (And XXI) was obtained with the Large Binocular Cameras at the Large Binocular Telescope. We have identified 50 variables in And XXI, of which 41 are RR Lyrae stars (37 fundamental-mode RRab, and 4 first-overtone -RRc, pulsators) and 9 are Anomalous Cepheids (ACs). The average period of the RRab stars $(\langle P a b\rangle=0.64$ days) and the period-amplitude diagram place And XXI in the class of Oosterhoff II - Oosterhoff-Intermediate objects. From the average luminosity of the RR Lyrae stars we derived the galaxy distance modulus of $(\mathrm{m}-\mathrm{M})_{0}=24.40 \pm 0.17 \mathrm{mag}$, which is smaller than previous literature estimates, although still consistent with them within $1 \sigma$. The galaxy color-magnitude diagram shows evidence for the presence of three different stellar generations in And XXI: 1$)$ an old $(\sim 12 \mathrm{Gyr})$ and metal poor $([\mathrm{Fe} / \mathrm{H}]=-1.7$ dex $)$ component traced by the RR Lyrae stars; 2) a slightly younger (10-6 Gyr) and more metal rich $([\mathrm{Fe} / \mathrm{H}]=-1.5$ dex) component populating the red horizontal branch, and 3$)$ a young age $(\sim 1 \mathrm{Gyr})$ component with same metallicity, that produced the ACs. Finally, we provide hints that And XXI could be the result of a minor merging event between two dwarf galaxies.
\end{abstract}

Subject headings: galaxies: dwarf, Local Group — galaxies: individual (Andromeda XXI) —stars: distances — stars: variables: other — techniques: photometric

\section{INTRODUCTION}

This is the second in our series of papers devoted to the study of Andromeda's (M31) satel-

\footnotetext{
* Based on data collected with the Large Binocular Cameras at the Large Binocular Telescope
}

lites based on time-series photometry obtained with the Large Binocular Cameras (LBC) of the Large Binocular Telescope (LBT). Our aim is to characterize the resolved stellar populations of the M31 companions using the color-magnitude diagram (CMD) and the properties of variable stars (see Clementini et al. 2011, for a general descrip- 
tion of the project). The final goal is to derive hints on the formation history of Andromeda's satellites and relate them to the global context of merging and accretion episodes occurring in M31. Details of the survey and results from the study of the M31 satellite Andromeda XIX (And XIX) were presented in Cusano et al. (2013, Paper I). In this paper we report on the study of another very extended M31 dwarf spheroidal (dSph) companion: Andromeda XXI (And XXI). The satellite galaxies of M31 are indeed an important test-bed for both the $\Lambda$ Cold Dark Matter $(\Lambda \mathrm{CDM})$ and the Modified Newtonian Dynamics (MOND, Milgrom 1983) theories. In the $\Lambda \mathrm{CDM}$ scenario the majority of the satellites are believed to be primordial dwarf galaxies residing in dark matter halos, which merge and are accreted to form larger galaxies (Zolotov et al. 2009). The satellites that we see today around M31 could thus be the residual building blocks of the M31 assembling process. However, some issues challenge the $\Lambda$ CDM scenario, among them is the phase-space distribution of the satellites around M31. Ibata et al. (2013) discovered that 15 of the more than 30 satellites of Andromeda lie in a thin plane $12 \mathrm{kpc}$ thick and over $200 \mathrm{kpc}$ in size, which rotates around M31. Accretion of small satellites through filaments has been invoked to explain the disky distribution of these M31 companions. However, the tiny width of the disk and the large number of satellites in it are features very hard to reproduce by $\Lambda \mathrm{CDM}$ simulations (see Pawlowski et al. 2014, for a complete discussion). A different explanation for the origin of the vast thin plane of satellites orbiting M31 is that they are tidal dwarf galaxies formed in the debris of a past major merger between M31 and a massive galaxy (Hammer et al. 2013). The space distribution of the satellites is well reproduced by numerical models of galaxy-interaction. A further alternative to the merger model is that the satellites formed during a past fly-by of the Milky Way (MW) and M31 about 10 Gyr ago. Indeed, under the assumption of Milgromiam dynamics 1 , Zhao et al. (2013) found that M31 and the MW had a close fly-by encounter between 7 and $11 \mathrm{Gyr}$ ago. Understanding the real nature/origin of the M31 satellites is thus crucial to address also cosmological theories.

1 Milgrom (1983)
And XXI was discovered by Martin et al. (2009) in the context of the PAndAS survey (Martin et al. 2013, and reference therein). The discovery paper reports a distance modulus of ( $\mathrm{m}$ $\mathrm{M})_{0}=24.67 \pm 0.13 \mathrm{mag}$ from the luminosity of the red giant branch tip and a half-light radius $\left(\mathrm{r}_{h}\right)$ of $3.5^{\prime}$ (corresponding to $r_{h}=875 \pm 127 \mathrm{pc}$ at the distance of And XXI). This makes And XXI the fourth largest dSph in the Local Group (LG). The galaxy has a metallicity of $[\mathrm{Fe} / \mathrm{H}]=-1.8 \pm 0.4$ dex, as estimated by Collins et al. (2013) from the calcium triplet (CaII) of the galaxy red giants. The same authors measured a velocity dispersion of $\sigma=4.5_{-1.0}^{+1.2} \mathrm{kms}^{-1}$ from 32 spectroscopically confirmed members, which is rather low, when compared with the great extension of And XXI. This was interpreted by Collins et al. (2013) as the result of a possible past tidal interaction with M31, however, for McGaugh \& Milgrom (2013) the low dispersion naturally arises in the MOND context. Conn et al. (2012) adopting a bayesian approach to locate the galaxy RGB tip have revised And XXI distance modulus to $(\mathrm{m}-\mathrm{M})_{0}=$ $24.59_{-0.07}^{+0.06} \mathrm{mag}$ which, although shorter, is still within $1 \sigma$ of Martin et al. (2009)'s value.

The paper is organized as follows. Observations, data reduction and calibration of And XXI photometry are presented in Section 2. Results on the identification and characterization of the variable stars, the catalog of light curves, and the Oosterhoff classification of And XXI are discussed in Sections 3. The distance to And XXI derived from the RR Lyrae stars is presented in Section 4. The galaxy CMD is discussed in Section 5 and the projected distribution of And XXI stellar components is Section 6. Section 7 gives some hints on the possibility of a past merging. Finally, a summary of the main results is presented in Section 8.

\section{OBSERVATIONS AND DATA RE- DUCTION}

Time series photometry in the $B$ and $V$ bands of a $23^{\prime} \times 23^{\prime}$ region centered on And XXI (R.A.= $23^{h} 54^{m} 47.7^{s}$, Decl. $=+42^{\circ} 28^{\prime} 15^{\prime \prime}$, J2000.0; Martin et al. 2009) was obtained in October and December 2010 with the LBC at the foci of the LBT. Sub-arcsec seeing conditions were achieved for the December observations, while seeing was 
Table 1: Log of And XXI observations

\begin{tabular}{lcccc}
\hline \hline Dates & Filter & $\mathrm{N}$ & $\begin{array}{c}\text { Exposure time } \\
(\mathrm{s})\end{array}$ & $\begin{array}{c}\text { Seeing (FWHM) } \\
(\operatorname{arcsec})\end{array}$ \\
\hline October 8-11, 2010 & $B$ & 6 & 420 & 1.4 \\
December 1-3, 2010 & $B$ & 40 & 420 & 0.8 \\
& & & & \\
October 8-11, 2010 & $V$ & 7 & 420 & 1.4 \\
December 1-3, 2010 & $V$ & 39 & 420 & 0.8 \\
\hline
\end{tabular}

slightly worse during the October run. Observations in the $B$ band were obtained with the Blue camera of the LBC, whereas the $V$ images were acquired with the Red camera. A total of $46 \mathrm{~B}$ and $46 \mathrm{~V}$ images each of 420 s exposure were obtained for a total exposure time of 19320s in each band. Images in both bands were dithered by 30 arcsec in order to fully cover the interCCD gaps of the LBC mosaic. Observations of And XIX were obtained in the same nights by interchanging the two targets in order to evenly sample the light curves of variable stars possibly occurring in the two galaxies. The log of the observations of And XXI is provided in Table 1 Data reduction was performed in the same way as for And XIX and is described in Paper I, to which the interested reader is referred for details. The PSF photometry was performed using the DAOPHOT-ALLSTAR-ALLFRAME package (Stetson 1987, 1994). Since the observations of And XXI were acquired within a few minutes from those of And XIX, for the absolute photometric calibration we used the calibrating equations derived in Paper I, by properly accounting for differences in airmass between the two targets.

\section{VARIABLE STARS}

Identification of the variable stars was performed using the variability index computed in DAOMASTER (Stetson 1994), then the light curves of the candidate variables were analyzed with the Graphical Analyzer of Time Series (GRATIS), custom software developed at the Bologna Observatory by P. Montegriffo (see, e.g., Clementini et al. 2000). Further details on the search for variables and the analysis of the light curves can be found in Paper I. A total of 50 variable stars were identified both in the $B$ and $V$ band datasets. The properties of the variable stars detected in And XXI are summarized in Table 2 We named the variables with an increasing number starting from the galaxy center, for which we adopted the coordinates by Martin et al. (2009). Column 1 gives the star identifier, Columns 2 and 3 provide the right ascension and declination (J2000 epoch), respectively. These coordinates were obtained from our astrometrized catalogs. Column 4 gives the type of variability. Columns 5 and 6 list the period and the Heliocentric Julian Day of maximum light, respectively. Columns 7 and 8 give the intensity-weighted mean $B$ and $V$ magnitudes, while Columns 9 and 10 list the corresponding amplitudes of the light variation. Light curves are presented in Figure 1

\subsection{RR Lyrae stars}

We discovered a total of $41 \mathrm{RR}$ Lyrae stars in And XXI, of which 37 are RRab and 4 RRc pulsators. The average period of the 37 bona fide RRab stars is $\left\langle\mathrm{P}_{\mathrm{ab}}\right\rangle=0.64 \mathrm{~d}(\sigma=0.06 \mathrm{~d})$. Considering only RRab stars inside the galaxy half-light radius the average period becomes $\left\langle\mathrm{P}_{\mathrm{ab}}\right\rangle=0.63$ days ( $\sigma=0.05$ days, average on 22 stars). From the average period of the RRab stars, And XXI can be classified as an Oosterhoff II (Oo II)/ Osterhoff Intermediate (Oo Int) object (Oosterhoff 1939). However, since the r.m.s of the average period is rather large, in order to better investigate the Oosterhoff nature of And XXI we produced the histogram of the RR Lyrae periods. This is shown in Figure 2. A multi-Gaussian fit was performed to find the peak value of this distribution. We found two separate maxima, a first higher peak is at $\mathrm{P}_{1}=0.60 \mathrm{~d}$ with $\sigma=0.02 \mathrm{~d}$ and contains about $36 \%$ of the RR Lyrae stars, and a second one at $\mathrm{P}_{2}=0.68 \mathrm{~d}$ with $\sigma=0.03 \mathrm{~d}$ and about $24 \%$ of the 
Table 2: Identification and properties of the variable stars detected in And XXI

\begin{tabular}{|c|c|c|c|c|c|c|c|c|c|}
\hline Name & $\begin{array}{c}\alpha \\
(2000)\end{array}$ & $\begin{array}{c}\delta \\
(2000)\end{array}$ & Type & $\begin{array}{l}\mathrm{P} \\
\text { (days) }\end{array}$ & $\begin{array}{c}\text { Epoch }(\max ) \\
\text { JD }(-2455000)\end{array}$ & $\begin{array}{c}\langle B\rangle \\
(\mathrm{mag})\end{array}$ & $\begin{array}{c}\langle V\rangle \\
(\mathrm{mag})\end{array}$ & $\begin{array}{c}\mathrm{A}_{B} \\
(\mathrm{mag})\end{array}$ & $\begin{array}{c}\mathrm{A}_{V} \\
(\mathrm{mag})\end{array}$ \\
\hline V1 & $23: 54: 47.066$ & $+42: 28: 19.42$ & RRc & 0.3950 & 533.684 & 25.87 & 25.38 & 0.66 & 0.51 \\
\hline $\mathrm{V} 2$ & $23: 54: 47.688$ & $+42: 27: 16.38$ & RRab & 0.6085 & 533.658 & 25.96 & 25.57 & 1.00 & 1.29 \\
\hline V3 & $23: 54: 49.205$ & $+42: 29: 15.29$ & RRab & 0.5810 & 477.692 & 25.77 & 25.30 & 1.21 & 0.95 \\
\hline V4 & $23: 54: 46.450$ & $+42: 27: 09.43$ & $\mathrm{AC}$ & 1.2353 & 532.625 & 24.22 & 23.63 & 1.33 & 1.03 \\
\hline V5 & $23: 54: 46.788$ & $+42: 29: 24.59$ & RRab & 0.6708 & 533.608 & 25.74 & 25.22 & 0.99 & 0.77 \\
\hline V6 & $23: 54: 51.778$ & $+42: 27: 37.58$ & $\mathrm{AC}$ & 1.067 & 476.959 & 24.63 & 24.07 & 1.06 & 0.53 \\
\hline V7 & $23: 54: 49.066$ & $+42: 29: 24.25$ & RRab & 0.6132 & 532.665 & 25.84 & 25.38 & 1.11 & 0.78 \\
\hline V8 & $23: 54: 46.471$ & $+42: 29: 37.67$ & RRab & 0.8342 & 531.621 & 25.55 & 24.93 & 0.83 & 0.8 \\
\hline V9 & $23: 54: 42.230$ & $+42: 27: 49.18$ & RRc & 0.3869 & 532.718 & 26.02 & 25.52 & 0.60 & 0.37 \\
\hline V10 & $23: 54: 53.642$ & $+42: 27: 45.65$ & RRab & 0.6371 & 532.605 & 25.82 & 25.33 & 0.87 & 0.57 \\
\hline V11 & $23: 54: 39.698$ & $+42: 29: 12.73$ & RRab & 0.6210 & 533.745 & 25.82 & 25.21 & 0.96 & 0.81 \\
\hline V12 & $23: 54: 49.212$ & $+42: 30: 26.64$ & RRab & 0.6662 & 533.727 & 25.85 & 25.31 & 1.25 & 1.06 \\
\hline V13 & $23: 54: 40.649$ & $+42: 26: 34.55$ & $\mathrm{AC}$ & 1.138 & 533.754 & 24.53 & 24.09 & 1.66 & 1.34 \\
\hline V14 & $23: 54: 38.774$ & $+42: 29: 26.20$ & $\mathrm{AC}$ & 1.072 & 533.590 & 24.96 & 24.44 & 1.18 & 0.85 \\
\hline V15 & $23: 54: 52.061$ & $+42: 30: 34.56$ & $\mathrm{AC}$ & 1.715 & 477.670 & 24.15 & 23.63 & 1.27 & 0.99 \\
\hline V16 & $23: 54: 38.664$ & $+42: 26: 52.66$ & RRab & 0.643 & 533.510 & 25.78 & 25.31 & 1.30 & 1.15 \\
\hline V17 & $23: 54: 56.498$ & $+42: 30: 06.08$ & RRab & 0.5768 & 532.663 & 25.78 & 25.26 & 1.30 & 1.08 \\
\hline V18 & $23: 54: 36.979$ & $+42: 29: 18.24$ & RRab & 0.5785 & 533.691 & 25.80 & 25.30 & 1.25 & 1.00 \\
\hline V19 & $23: 54: 36.562$ & $+42: 27: 21.82$ & $\mathrm{AC}$ & 1.390 & 531.670 & 24.27 & 23.80 & 1.32 & 1.00 \\
\hline V20 & $23: 54: 52.507$ & $+42: 30: 56.59$ & RRab & 0.6193 & 532.710 & 25.99 & 25.41 & 0.83 & 0.64 \\
\hline V21 & $23: 54: 37.603$ & $+42: 30: 16.38$ & RRab & 0.5971 & 533.717 & 25.84 & 25.32 & 1.19 & 0.92 \\
\hline V22 & $23: 54: 54.931$ & $+42: 31: 04.19$ & RRab & 0.5911 & 532.740 & 25.87 & 25.36 & 0.78 & 0.63 \\
\hline V23 & $23: 54: 35.311$ & $+42: 29: 34.87$ & RRab & 0.6661 & 531.686 & 25.74 & 25.21 & 1.24 & 1.05 \\
\hline V24 & $23: 54: 59.700$ & $+42: 26: 26.59$ & RRab & 0.6019 & 531.690 & 25.87 & 25.37 & 1.08 & 0.79 \\
\hline V25 & $23: 54: 33.816$ & $+42: 27: 37.80$ & RRab & 0.6368 & 531.750 & 25.93 & 25.42 & 0.86 & 0.76 \\
\hline V26 & 23:55:01.913 & $+42: 28: 29.10$ & $\mathrm{AC}$ & 1.1500 & 480.518 & 24.05 & 23.58 & 1.19 & 0.94 \\
\hline V27 & 23:55:01.992 & $+42: 28: 35.47$ & RRab & 0.7052 & 480.660 & 25.60 & 25.04 & 0.59 & 0.46 \\
\hline V28 & $23: 54: 59.134$ & $+42: 26: 03.77$ & RRc & 0.3058 & 533.711 & 25.68 & 25.34 & 0.76 & 0.56 \\
\hline V29 & 23:55:01.531 & $+42: 27: 10.69$ & RRab & 0.6414 & 532.605 & 25.78 & 25.28 & 0.78 & 0.60 \\
\hline V30 & $23: 54: 45.857$ & $+42: 24: 38.95$ & RRab & 0.5898 & 531.768 & 25.74 & 25.28 & 1.33 & 1.12 \\
\hline V31 & $23: 55: 02.167$ & $+42: 27: 34.31$ & RRab & 0.5880 & 533.640 & 25.74 & 25.31 & 0.85 & 0.80 \\
\hline V32 & $23: 55: 01.817$ & $+42: 29: 23.64$ & RRab & 0.6513 & 533.530 & 25.79 & 25.38 & 1.12 & 0.86 \\
\hline V33 & $23: 54: 52.793$ & $+42: 24: 41.00$ & RRab & 0.6182 & 533.750 & 25.95 & 25.41 & 1.20 & 0.89 \\
\hline V34 & $23: 54: 39.821$ & $+42: 31: 39.79$ & $\mathrm{AC}$ & 1.2460 & 532.702 & 24.45 & 24.01 & 1.30 & 0.98 \\
\hline V35 & $23: 54: 44.129$ & $+42: 32: 06.32$ & RRab & 0.6061 & 532.770 & 25.92 & 25.31 & 1.13 & 0.88 \\
\hline V36 & $23: 54: 47.352$ & $+42: 32: 16.44$ & RRab & 0.6167 & 480.650 & 25.88 & 25.37 & 0.94 & 0.77 \\
\hline V37 & $23: 54: 34.003$ & $+42: 30: 25.91$ & RRab & 0.7074 & 532.768 & 25.77 & 25.26 & 0.84 & 0.79 \\
\hline V38 & $23: 54: 57.665$ & $+42: 31: 48.01$ & RRc & 0.2851 & 532.700 & 25.89 & 25.45 & 0.63 & 0.39 \\
\hline V39 & $23: 54: 35.669$ & $+42: 31: 30.14$ & RRab & 0.5707 & 477.705 & 25.87 & 25.38 & 1.02 & 0.91 \\
\hline V40 & $23: 54: 47.770$ & $+42: 23: 10.75$ & RRab & 0.7178 & 532.760 & 25.77 & 25.28 & 0.82 & 0.64 \\
\hline V41 & $23: 54: 45.545$ & $+42: 33: 18.90$ & RRab & 0.5860 & 531.715 & 25.89 & 25.45 & 1.24 & 0.87 \\
\hline V42 & $23: 54: 39.228$ & $+42: 23: 22.13$ & RRab & 0.6824 & 531.748 & 25.70 & 25.21 & 1.17 & 0.85 \\
\hline V43 & $23: 54: 36.629$ & $+42: 33: 24.98$ & RRab & 0.6078 & 531.747 & 25.88 & 25.35 & 1.16 & 0.91 \\
\hline V44 & $23: 55: 04.075$ & $+42: 32: 50.46$ & $\mathrm{AC}$ & 1.1655 & 477.405 & 24.21 & 23.82 & 1.12 & 0.87 \\
\hline V45 & $23: 54: 43.080$ & $+42: 21: 30.78$ & RRab & 0.5670 & 533.650 & 25.80 & 25.34 & 1.17 & 1.03 \\
\hline V46 & $23: 55: 14.446$ & $+42: 31: 01.42$ & RRab & $0.71:$ & 533.726 & 25.89 & 25.30 & 0.81 & 0.63 \\
\hline V47 & $23: 55: 16.555$ & $+42: 28: 51.39$ & RRab & 0.5646 & 532.655 & 25.84 & 25.34 & 1.51 & 1.17 \\
\hline V48 & $23: 55: 21.782$ & $+42: 29: 46.14$ & RRab & 0.7560 & 532.795 & 25.83 & 25.36 & 0.68 & 0.61 \\
\hline V49 & $23: 53: 54.898$ & $+42: 25: 56.04$ & RRab & 0.6766 & 532.652 & 25.79 & 25.29 & 0.94 & 0.73 \\
\hline V50 & $23: 55: 46.274$ & $+42: 26: 55.86$ & RRab & 0.6920 & 531.762 & 25.84 & 25.39 & 0.80 & 0.57 \\
\hline
\end{tabular}



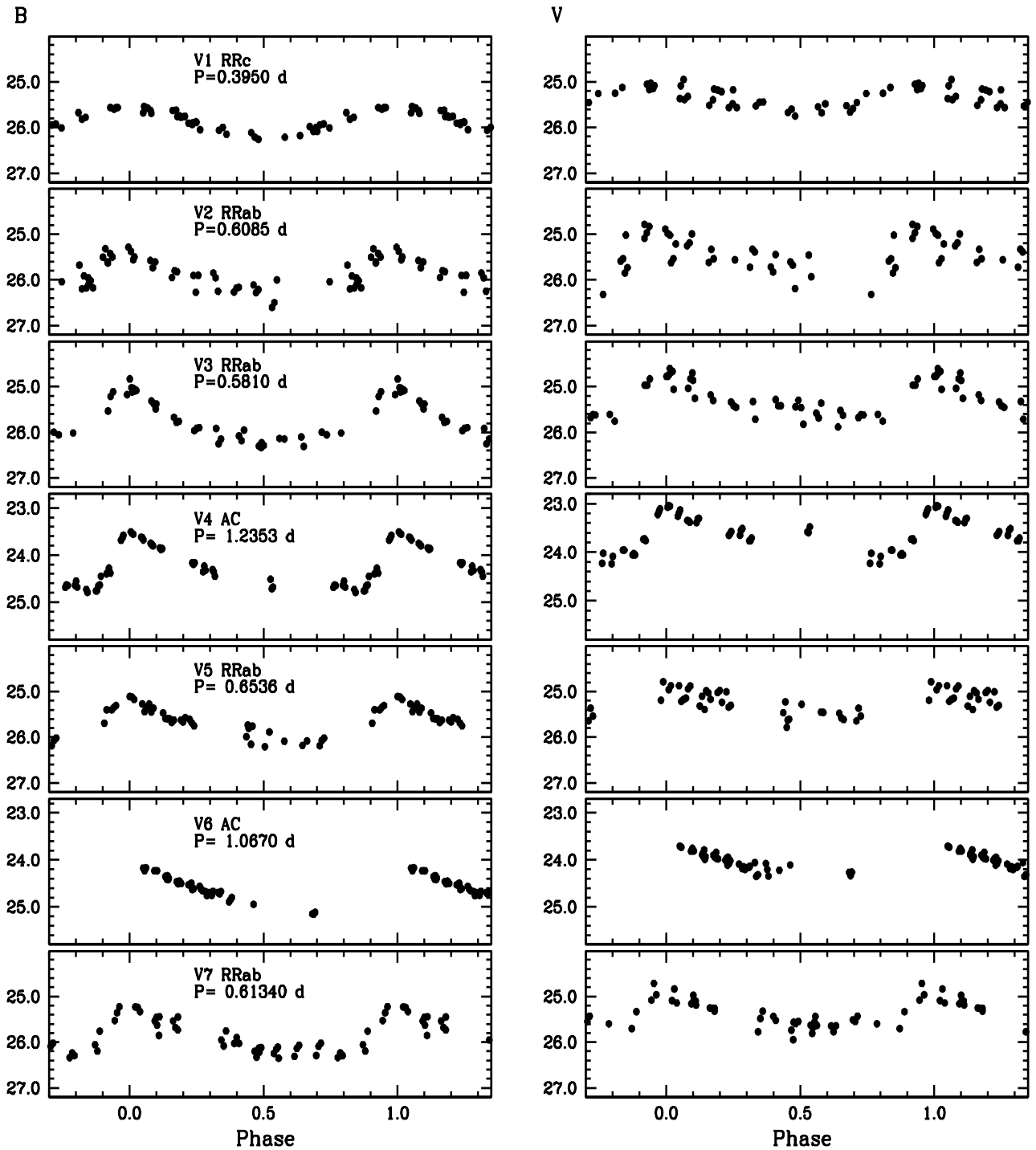

Fig. 1.-B (left panels) and $V$ (right panels) light curves of the variable stars identified in And XXI. Stars are ordered with increasing the distance from the galaxy center, for which we adopted Martin et al. (2009) coordinates. Typical internal errors for the single-epoch data are in the range of 0.02 to 0.18 mag in $B$, and of 0.03 to 0.25 mag in $V$. 
B
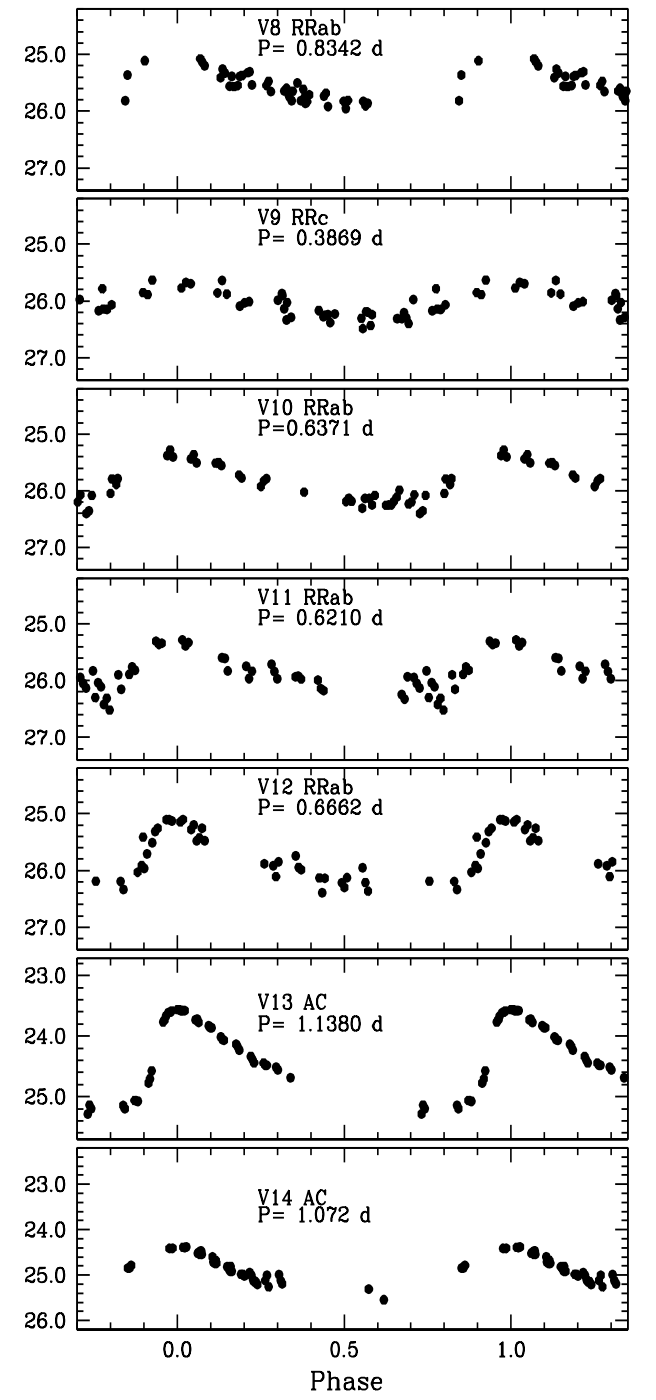

V

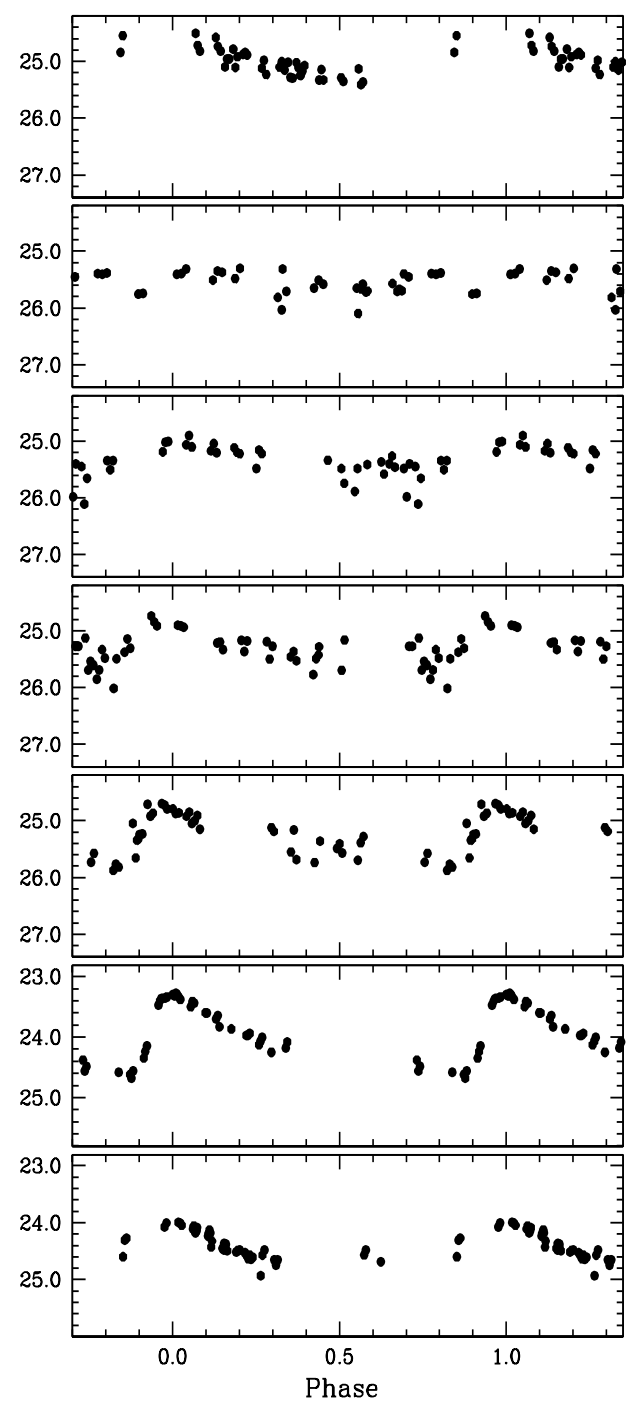

Fig. 1.- continued - 

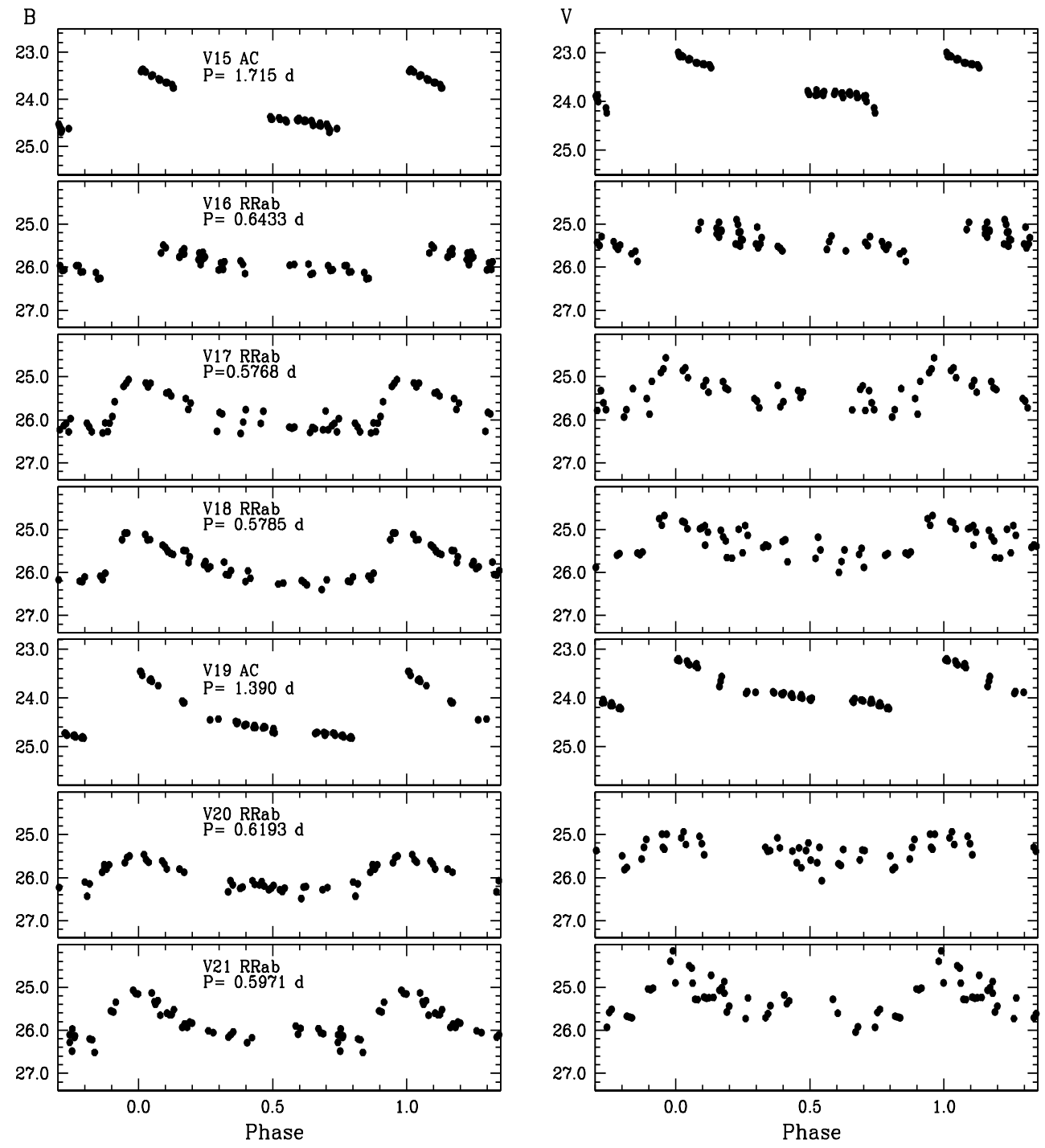

Fig. 1. - continued - 
B
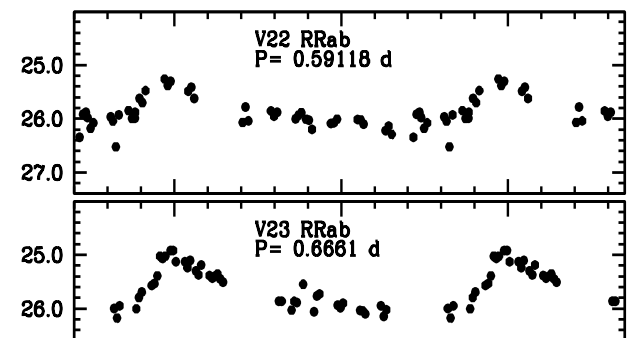

27.0
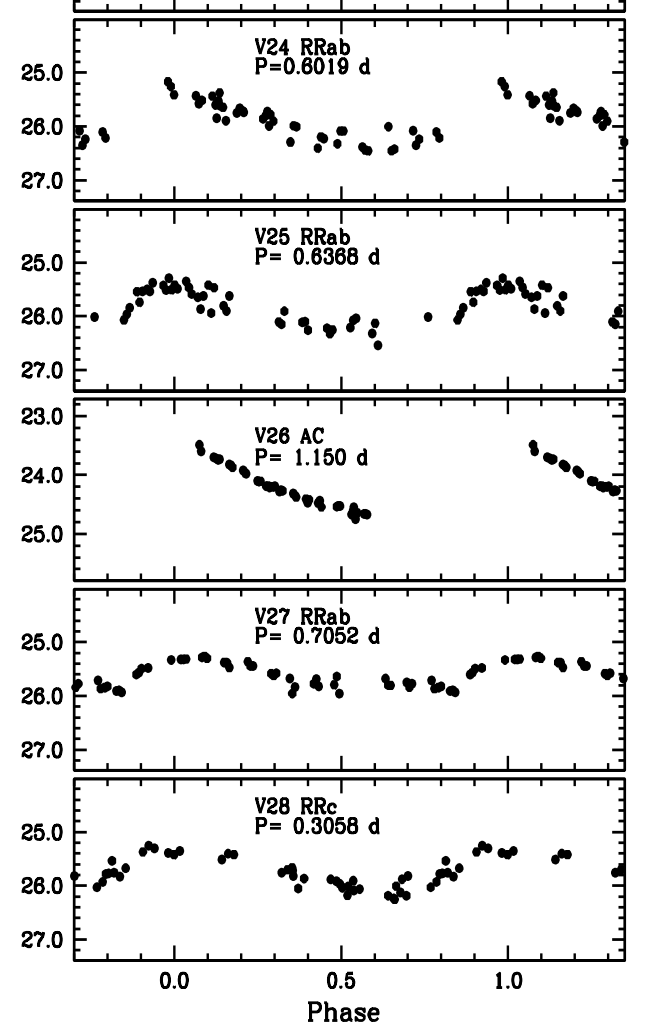

V
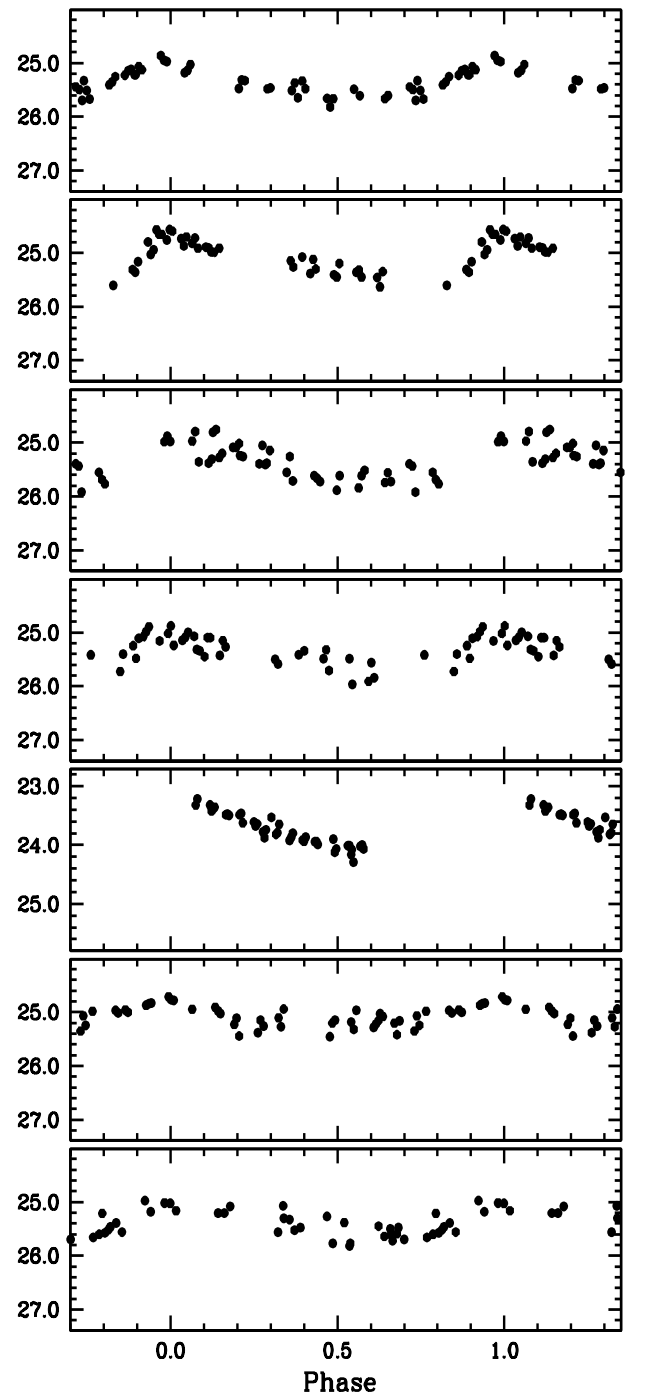

Fig. 1.- continued - 
B
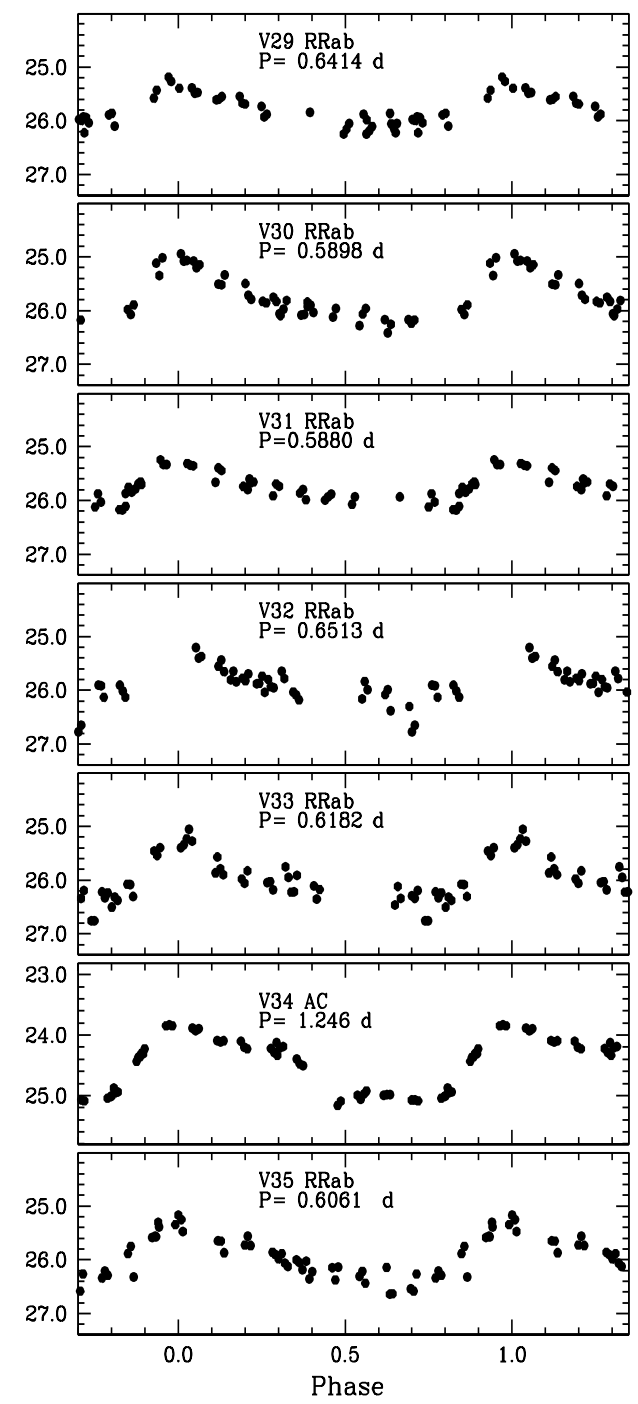

V

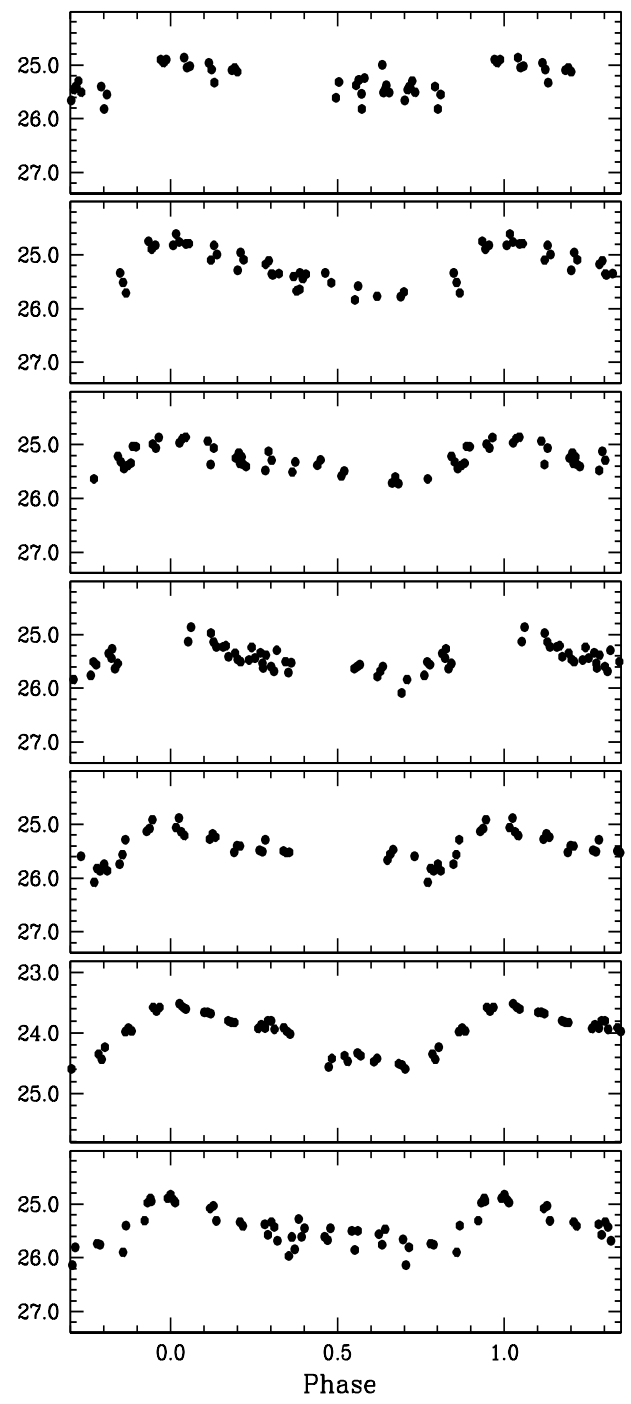

Fig. 1.- continued - 
B

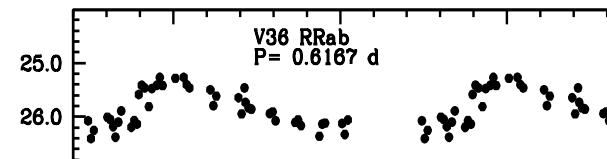

27.0
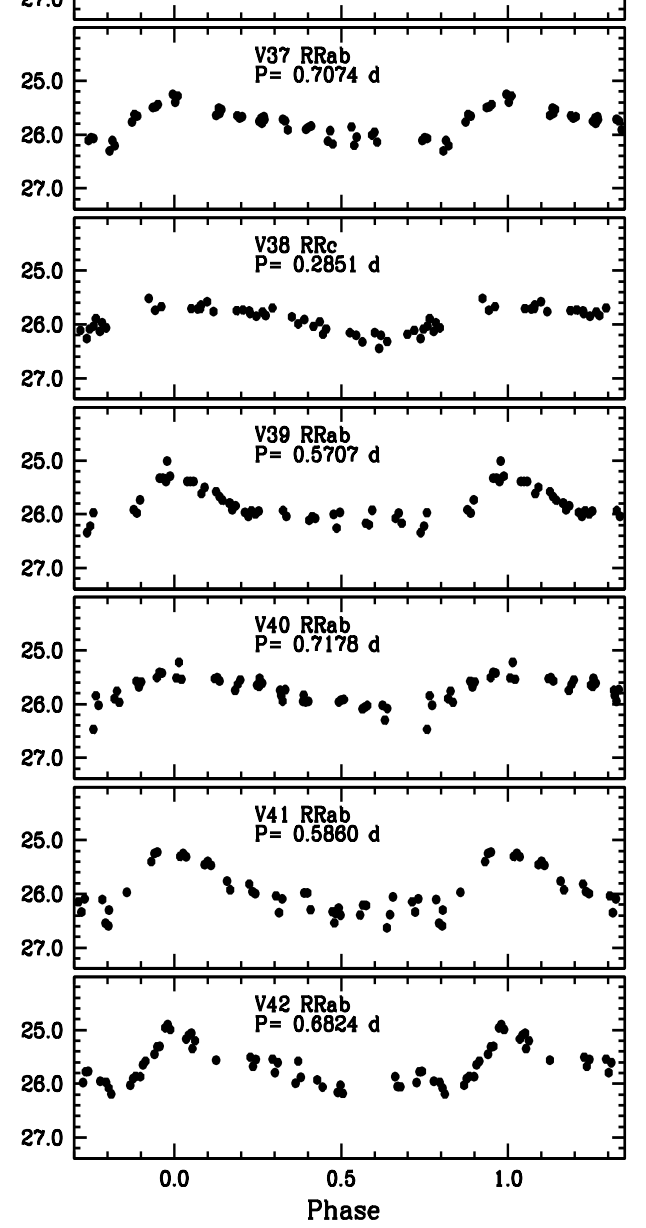

V
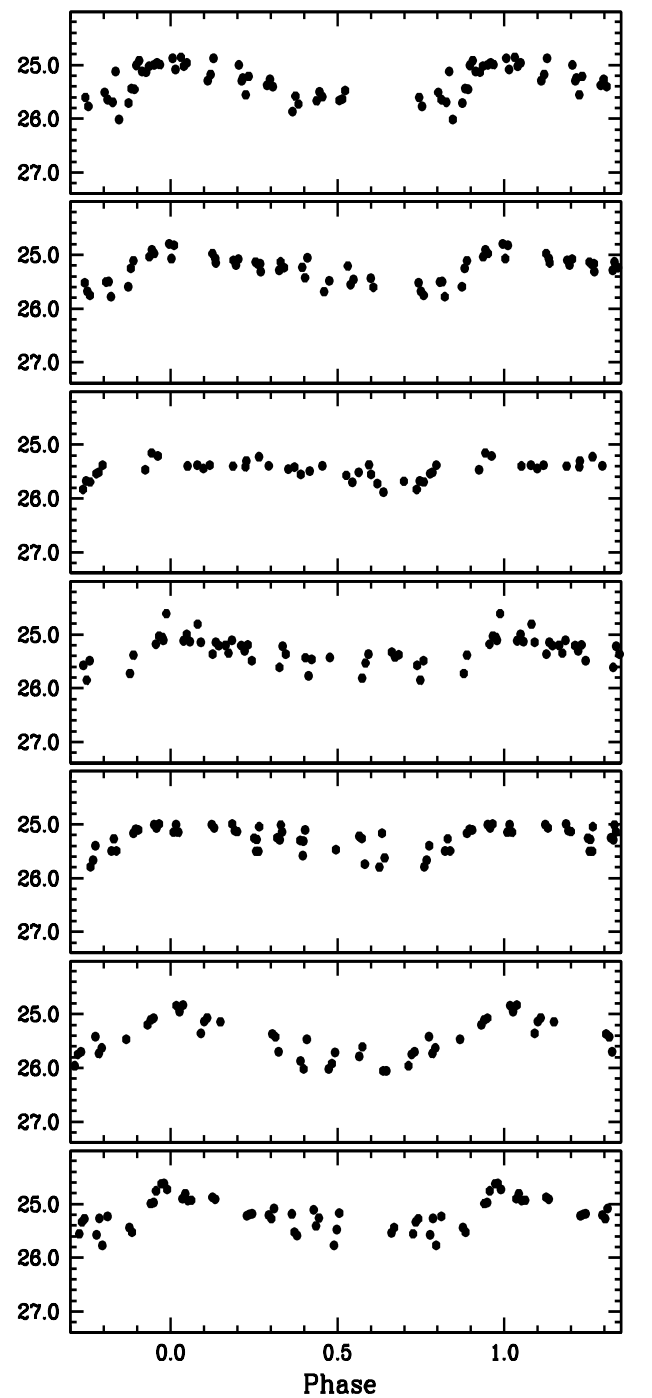

Fig. 1.- continued - 
B

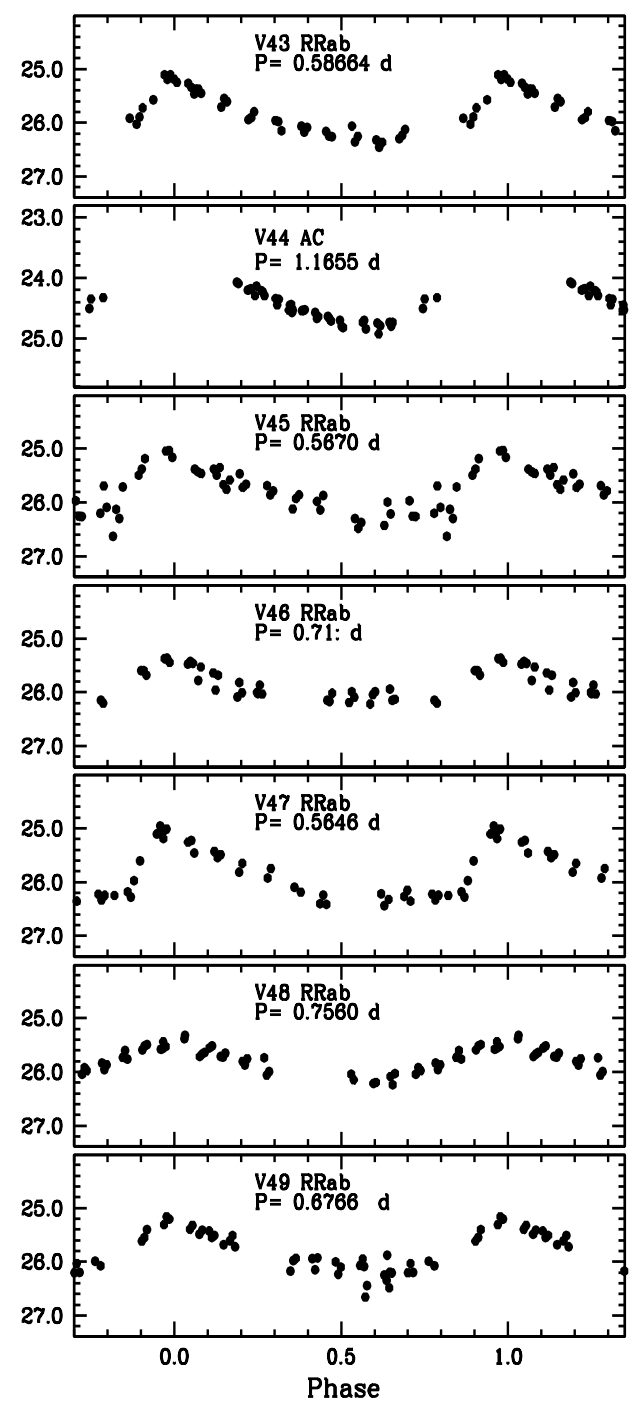

V

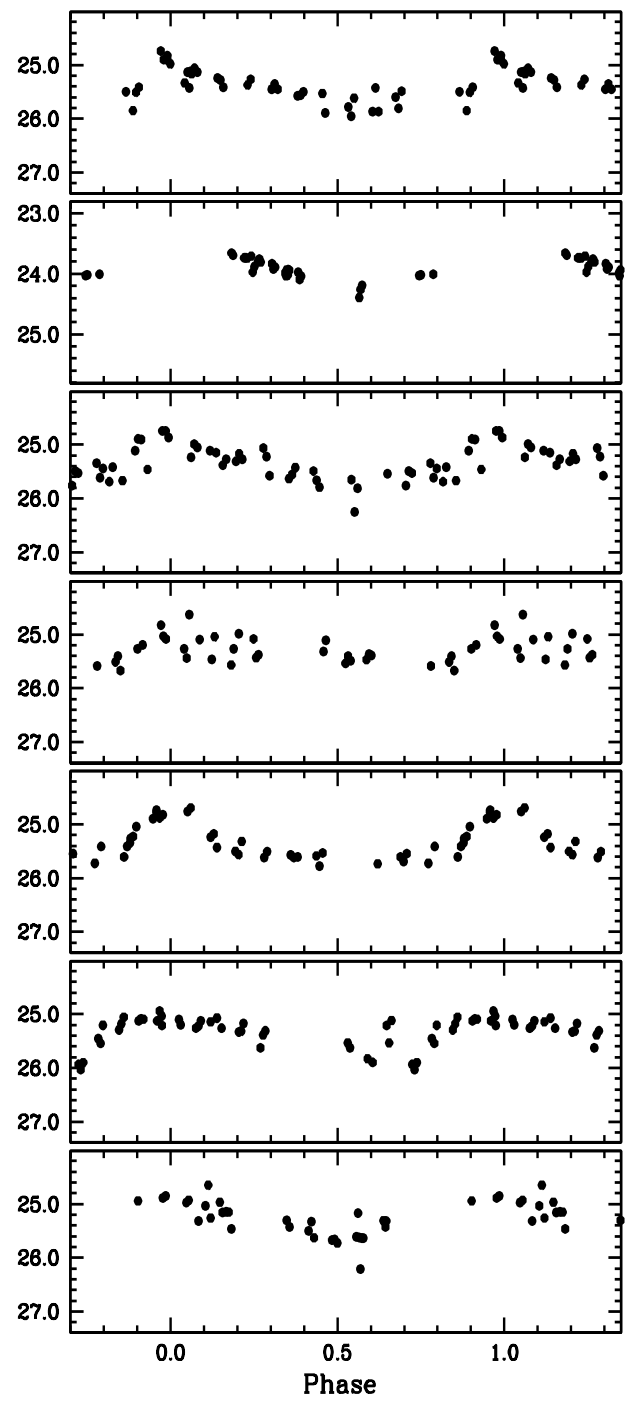

Fig. 1.- continued - 

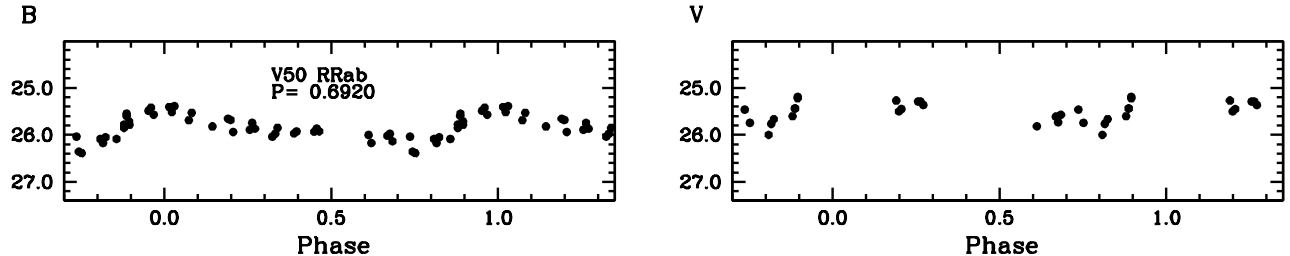

Fig. 1. - continued -

variables. The fit is shown in Figure 2, The presence and relative strength of these two peaks reinforces for And XXI a classification as Oo Int/Oo II object. In the histogram two RR Lyrae stars, V8 and V48, show longer periods when compared to the average distribution. We comment on these stars further in Section 3.3 .

The period-amplitude diagram (also known as Bailey diagram, Bailev 1902) of the And XXI RR Lyrae stars is shown in Figure 3 together with the loci defined by bona-fide regular (solid line) and the well-evolved (dashed line) RR Lyrae stars in the Galactic globular cluster M3, according to Cacciari et al. (2005). M3 regular RR Lyrae stars have Oosterhoff I (Oo I) properties, while the M3 evolved variables mimic the location of the Oo II variables. The majority of the And XXI RR Lyrae stars fall between the two loci and near the position of the Oo II locus, thus confirming the galaxy classification as an Oo Int/Oo II object. The fraction of RRc stars over the total number of RR Lyrae stars is $\mathrm{f}_{c}=\mathrm{N}_{c} / \mathrm{N}_{\mathrm{ab}+\mathrm{c}}=0.10$, that is small both for an Oo II $\left(\mathrm{f}_{c} \sim 0.44\right)$ and an Oo I $\left(\mathrm{f}_{c} \sim 0.17\right)$ system. This fraction is also small compared to other dwarf galaxies of similar metallicity $(\mathrm{Fe} / \mathrm{H}=-1.8 \mathrm{dex}$, Collins et al. 2013) like for example And XIX $\left(\mathrm{f}_{c}=0.26\right.$, Paper I). RRc stars have smaller amplitudes, hence they are more difficult to identify than RRab stars. Furthermore, they have bluer colors. Hence, as it will be discussed in Section 5, they occur in the region of the CMD which is heavily contaminated by background galaxies. This could affect our capability of detecting RRc stars. However, we individually checked for variability all stars bluer than $B-V \sim 0.40$ mag in the HB region of the CMD and

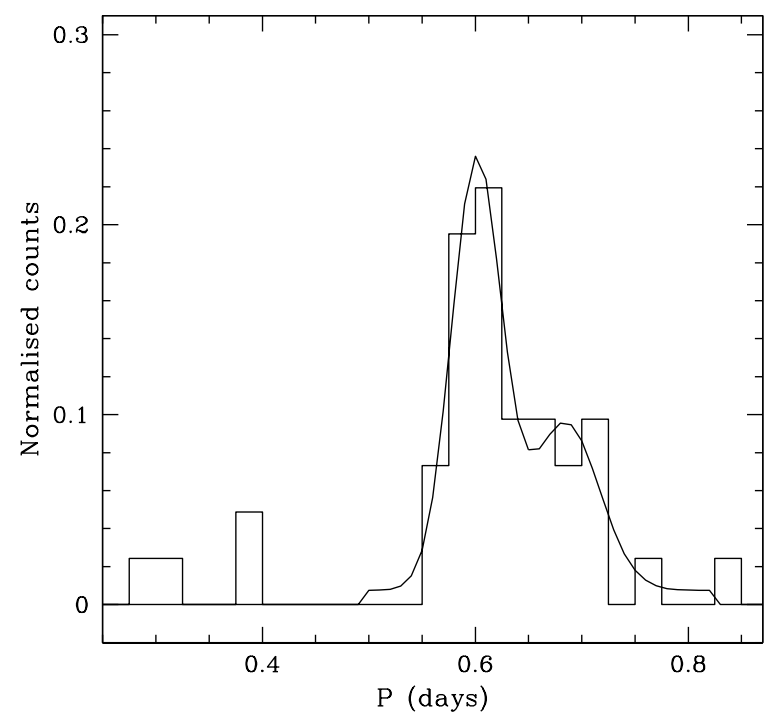

Fig. 2.- Histogram of the periods of the RR Lyrae stars identified in And XXI. The bin size is 0.025 days. The gaussian fit to the period histogram is also shown. The two stars at $\mathrm{P}=0.8342$ $\mathrm{d}$ and $\mathrm{P}=0.756 \mathrm{~d}$ are $\mathrm{V} 8$ and $\mathrm{V} 48$, respectively. 


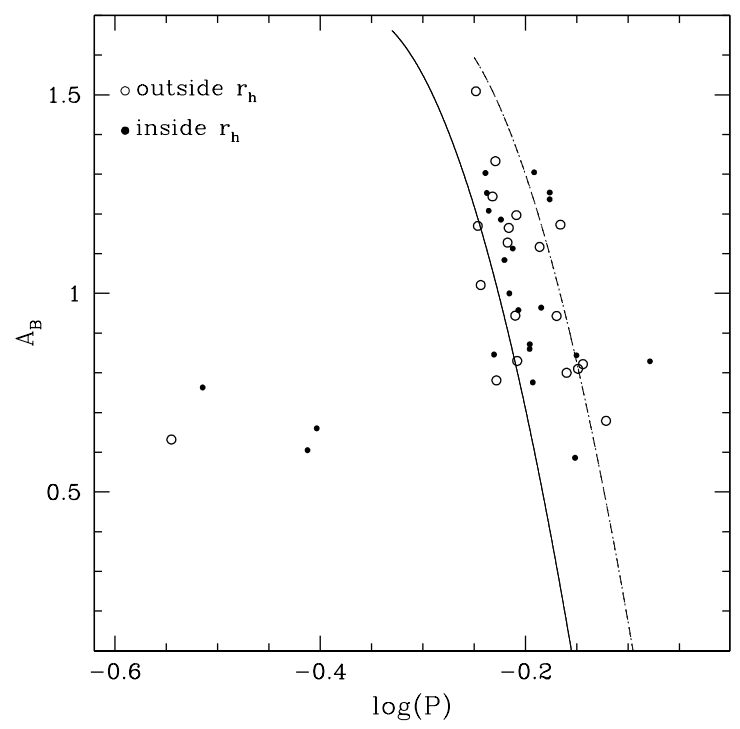

Fig. 3.- Period-amplitude diagram for the RR Lyrae stars in And XXI. Solid and dashed lines represent the loci defined by bona-fide regular and well-evolved RR Lyrae stars in M3 (Cacciari et al. 2005), respectively. Filled and open symbols mark variables within and outside the galaxy half-light radius, respectively.

did not find additional variables. Furthermore, in And XIX as well as in other M31 dSphs we are studying, we do find RRc stars, in spite of the contamination by background galaxies in that part of the CMD. Hence, we are inclined to think that the paucity of first overtone pulsators in And XXI is a real feature.

The spatial distribution of the RR Lyrae stars is shown in Figure 4 (filled red circles), and indicates a rather complex structure for And XXI. The RR Lyrae stars seem to be asymmetrically distributed. In particular, outside the galaxy halflight radius we note two elongated features traced by the variable stars, one $\sim 12$ arcmin long going from North-West to South-East and the other pointing towards South. They may trace past interactions with a dwarf satellite galaxy of M31 and/or with M31 itself. We discuss this more in detail in Section 5 .

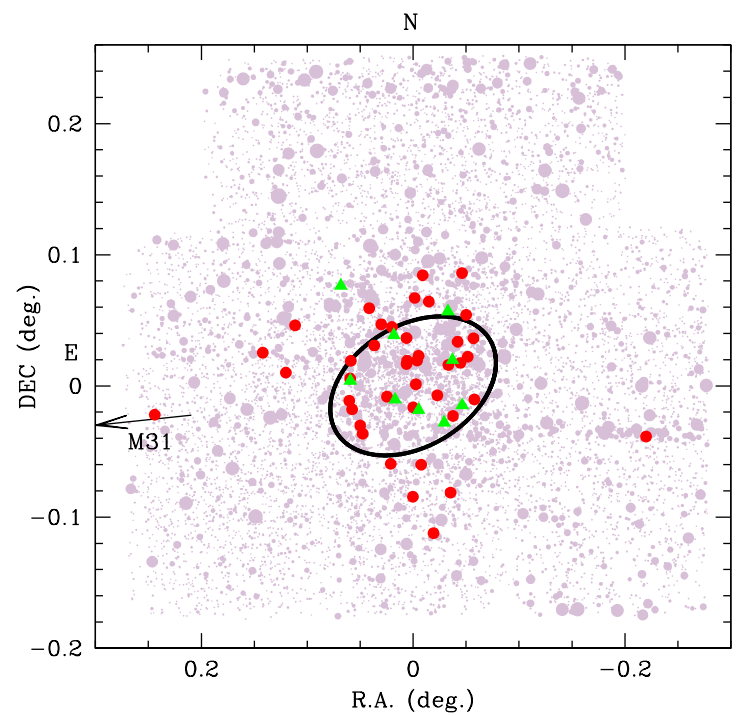

Fig. 4.- Spatial distribution of the variable stars detected in And XXI. Red filled circles mark the RR Lyrae stars and green filled triangles are the ACs. Symbol size for all other stars is proportional to the magnitude. An ellipse marks the area enclosed in the half-light radius defined by Martin et al.(2009). The arrow marks the direction towards the M31 center. 


\subsection{Anomalous Cepheids}

We found in And XXI nine variable stars which are $\sim 1$ mag brighter than the average magnitude of the RR Lyrae stars. We classified these stars as ACs based on the comparison with stellar isochrones and the period-Wesenheit $(P W)$ relation of ACs. The Wesenheit function (van den Bergh 1975; Madore 1982) is defined as: $\mathrm{W}(B, V)=\mathrm{M}_{V}-3.1 \times(B-V)$, where $\mathrm{M}_{V}$ is the $V$ magnitude corrected for the distance. The $\langle V\rangle$ magnitudes of the ACs were corrected adopting the distance modulus $(\mathrm{m}-\mathrm{M})_{0}=24.40 \pm 0.17 \mathrm{mag}$ derived from the RR Lyrae stars (see Section 4) and used to derive the corresponding Wesenheit indices. The $P W$ relations of And XXI ACs are shown in the left panel of Figure 5 along with the $P W$ relation for Fundamental (F) mode and First Overtone (FO) mode ACs recently derived for the Large Magellanic Cloud (LMC, solid lines) by Ripepi et al. (2014)2. The nine ACs in And XXI well follow, within the errors, the Ripepi et al. (2014) relations for F and FO pulsators. To confirm that these bright variables are mostly ACs, in the right panel of Figure [5 we compare them with the $P W$ relation for Classical Cepheids (CCs) in the LMC derived by Soszynski et al. (2008). The $P W$ relations for CCs indeed do not fit very well the bright variables in And XXI. However, we cannot totally reject the possibility that at least one, perhaps two of the bright variables (namely, V4 and V26) could be CCs. As in Paper I we derived the specific frequency of ACs in the galaxy, on the assumption that all the 9 bright variables in And XXI are bona fide ACs. This is plotted in Figure 6 along with the AC specific frequency in a number of MW and M31 dwarf satellites. And XXI (starred symbol in Figure 6) well follows the relation traced by the other dwarf galaxies. The error is computed considering a Poisson statistics and include the case in which 2 of the ACs are in fact CCs.

\subsection{Comments on individual peculiar stars}

V2 - The star is 0.3 mag fainter than the average $V$ magnitude of RR Lyrae stars. The pulsation amplitude in the $V$ band is larger than in the $B$ band and the $\mathrm{V}$ light curve although well sampled is very noisy. The color of the star is blue ( $B-$ $V)=0.39 \mathrm{mag}$ ) for an RRab. Only V28 that is an RRc star is bluer. V2 is likely a RR Lyrae star of the M31 field.

V4 - The $V$ light curve is sparsely sampled between phase 0.40 and 0.70 . However, maximum and minimum light are well covered, hence the intensity-averaged mean $V$ magnitude should be correct. The star is about 1.5 magnitude brighter than the horizontal branch and in the $P W$ plane V4 falls within $1 \sigma$ from the relation for first overtone ACs, but also fits well the relation for fundamental-mode CCs.

V6 - Due to the star pulsation period $(\mathrm{P}=1.067$ d) and our data sampling, we do not have data points in the rising part of the light curve both in $B$ and $V$. This affects the star mean magnitudes. In fact in the $P W$ plane V6 is slightly off the relation for FO ACs.

V8 - This is the longest period $(\mathrm{P}=0.8342 \mathrm{~d})$ and the brightest ( $V=24.93 \mathrm{mag}$ ) RR Lyrae in our sample. It has very similar amplitudes in $B$ and $V$. V8 is also the reddest variable in And XXI. In Figure 5 V8 is plotted with a star sign, and shows to follow well the $P W$ relation for F-mode ACs, suggesting that this star could be an AC.

V26 - As for V6 this AC lacks part of the $B$ and $V$ light curves due to the combination of pulsation period $(\mathrm{P}=1.1500 \mathrm{~d})$ and sparse data sampling.

V27 - This is one of the two brightest RR Lyrae stars in our sample. The $B$ and $V$ amplitudes are small for a RRab of that period ( $\mathrm{P}=0.7052$ days), nevertheless the amplitude ratio $\mathrm{A}_{B} / \mathrm{A}_{V}=1.28$ is typical of a RR Lyrae star.

V46 - The star period is uncertain and the light curves are noisy. However amplitudes and average magnitudes are typical of a normal RR Lyrae star.

V49/V50- These two variables are about $12^{\prime}$ away from the galaxy center. However, the pulsation period and the mean magnitudes are consistent with the average values of the RR Lyrae stars in And XXI. We will show is Section 6 that portions of And XXI seem to extend that far from the galaxy center.
${ }^{2}$ Ripepi et al. (2014)'s relations were derived for the $V$ and $I$ bands, and we have converted them to $B$ and $V$ using equation 12 of Marconi et al. (2004). 


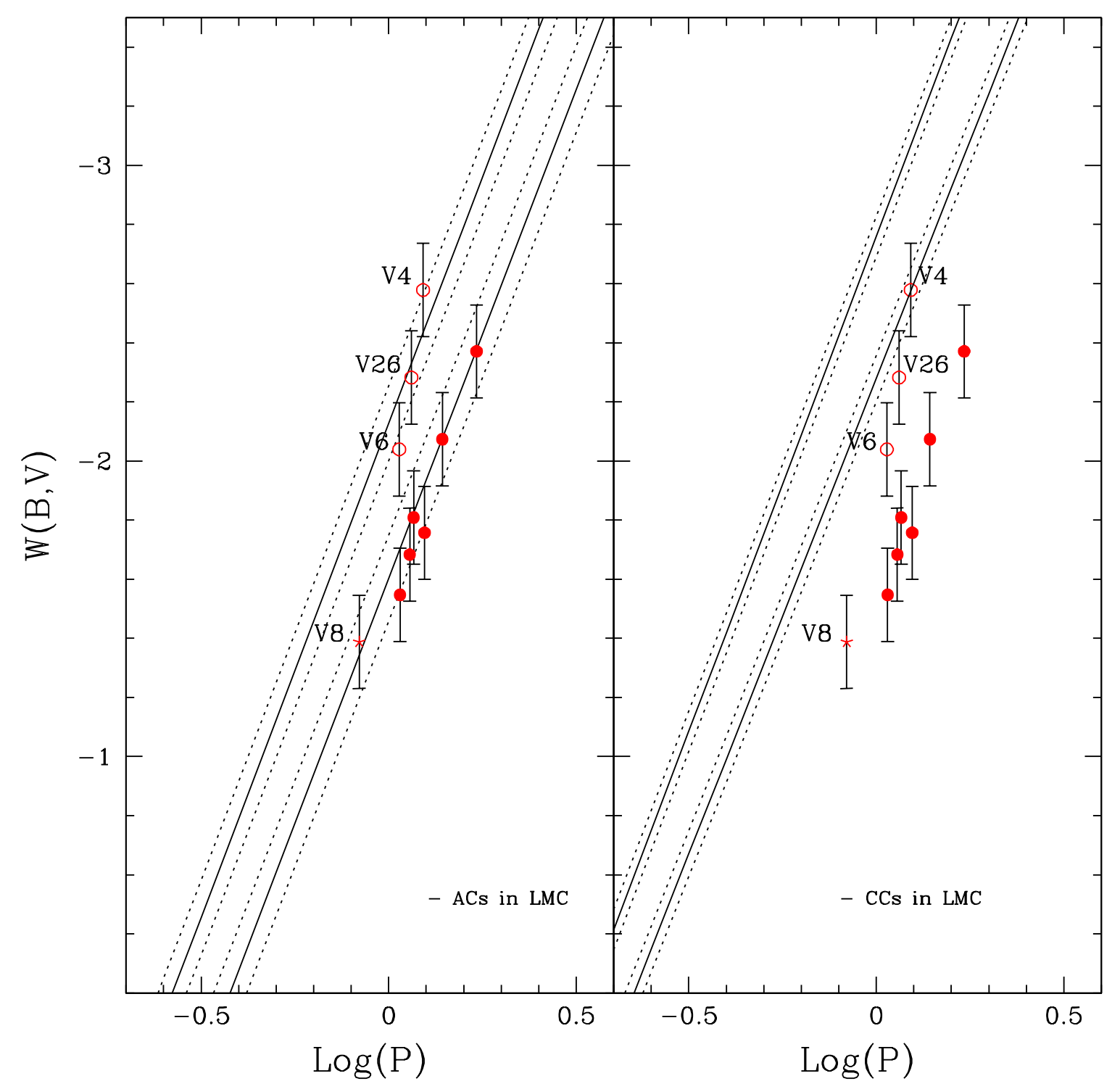

Fig. 5.- $P W$ relations for the 9 variable stars in And XXI which are brighter than the horizontal branch. Red filled circles are fundamental mode pulsators, open red circles are first-overtone variables. Also plotted as a star is the bright RR Lyrae variable V8 (see details in the text). Solid lines in the left panel are the $P W$ relations for ACs in the LMC (Ripepi et al. 2014), while on the right panel are shown the $P W$ relations for CCs in the LMC from Soszynski et al. (2008). Dashed lines show the $1 \sigma$ contours. 


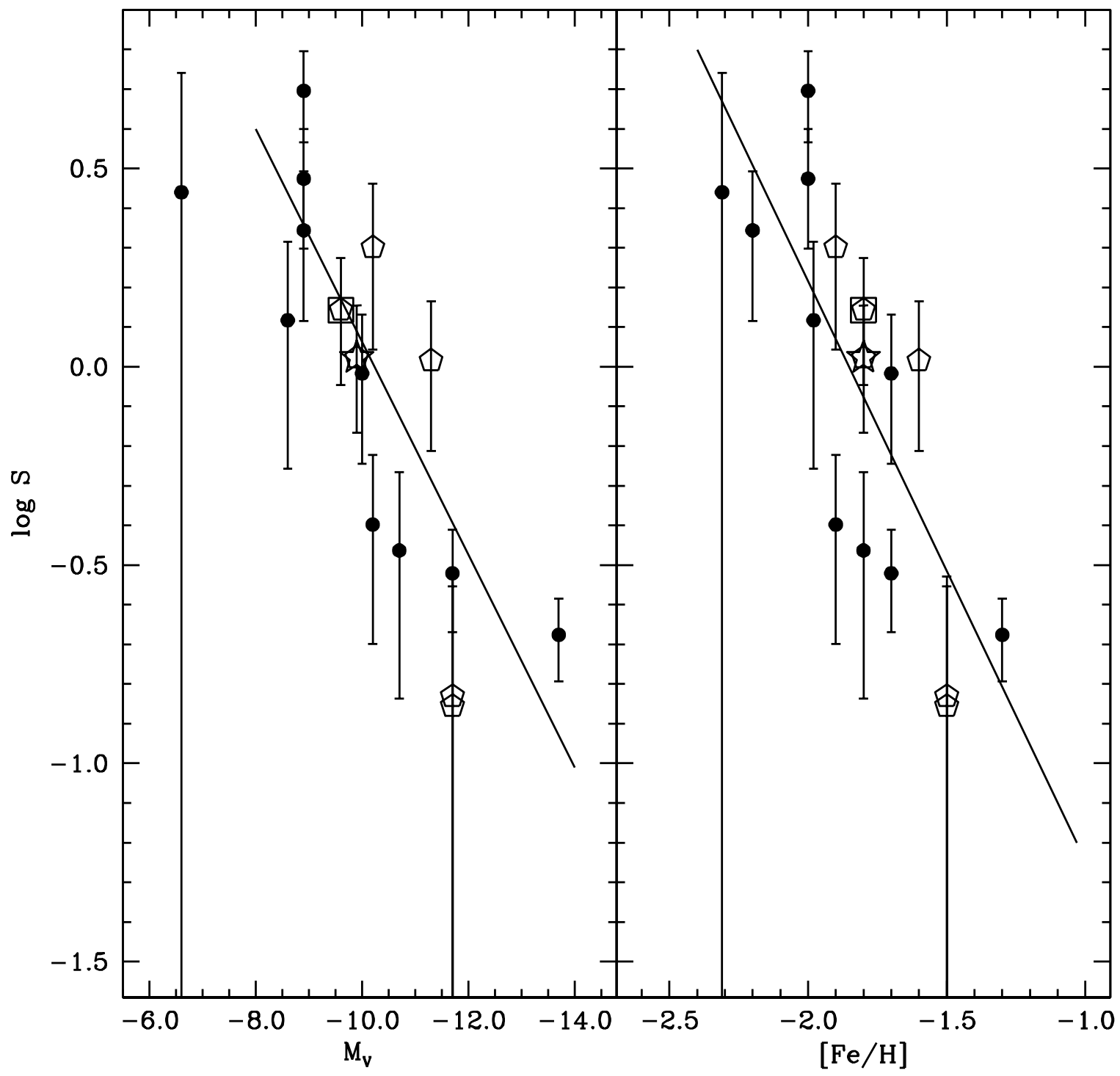

Fig. 6. - Left: Specific frequency of ACs in dwarf satellites of the MW (filled circles) and M31 (open pentagons) versus absolute visual magnitude. And XXI is represented by a starred symbol, And XIX by an open square. The black lines in both panels are the best fits to the data. Right: Same as in the left panel, but versus metallicity of the parent galaxy. 


\section{DISTANCE}

The distribution of the intensity-averaged mean $V$ magnitudes of the RR Lyrae stars in And XXI is shown in the histogram of Figure 7 The average magnitude of the $41 \mathrm{RR}$ Lyrae stars in And XXI is $\langle V(R R)\rangle=25.33 \pm 0.11 \mathrm{mag}$ (average on 41 stars). Considering only RR Lyrae stars inside the area defined by the galaxy $\mathrm{r}_{h}$, the average becomes $\langle V(R R)\rangle=25.32 \pm 0.13 \mathrm{mag}$ (average on 22 stars). Among the RR Lyrae stars V8 and V27 are 0.3 mag brighter than the RR Lyrae average $V$ magnitude and are most probably blended objects or foreground RR Lyrae stars of the M31 halo. On the faint side variables V2 and V9 are $\sim 0.3 \mathrm{mag}$ fainter than the RR Lyrae average $V$ magnitude and possibly are RR Lyrae belonging either to the M31 halo or to a structure around M31. Excluding these four stars (V2, V8, V9 and V27) the averages we obtain are $\langle V(R R)\rangle=25.33 \pm 0.06 \mathrm{mag}$ (average on 37 stars) for all and $\langle V(R R)\rangle=25.32 \pm 0.12$ mag (average on 19 stars) for the RR Lyrae stars inside the galaxy $\mathrm{r}_{h}$. We adopt the latter value for the average $V$ magnitude of And XXI RR

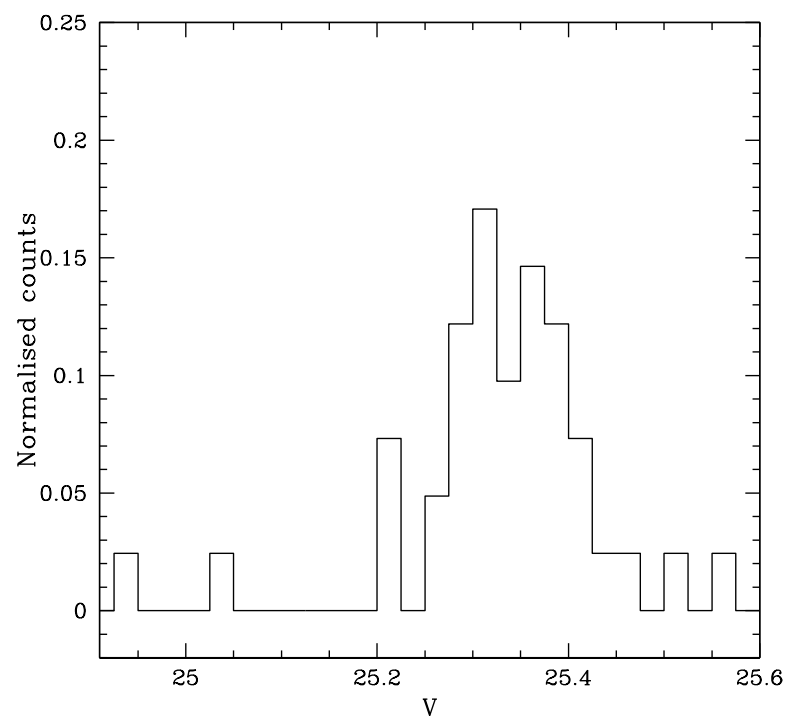

Fig. 7.- Histogram of the intensity-averaged mean $V$ magnitudes of the RR Lyrae stars identified in And XXI. The bin size is 0.025 mag. The two brightest stars are V8 and V27, the two faintest ones are V2 and V9.
Lyrae stars, $\mathrm{M}_{\mathrm{V}}=0.54 \pm 0.09 \mathrm{mag}$ for the absolute visual magnitude of RR Lyrae stars with metallicity of $[\mathrm{Fe} / \mathrm{H}]=-1.5 \mathrm{dex}$ Clementini et al. 2003 ) and $\frac{\Delta \mathrm{M}_{\mathrm{v}}}{\Delta \mid \mathrm{Fe} / \mathrm{H}}=0.214 \pm 0.047 \mathrm{mag} / \mathrm{dex}$ (Clementini et al. 2003; Gratton et al. 2004) for the slope of the RR Lyrae luminosity-metallicity relation. For And XXI metallicity we adopt the value $[\mathrm{Fe} / \mathrm{H}]=-1.8 \pm 0.4 \mathrm{dex}$ as derived spectroscopically by Collins et al. (2013). To correct for interstellar extinction we derived the reddening from the galaxy RR Lyrae stars using the method by Piersimoni et al. (2002). This is based on the relation between intrinsic $B-V$ color, period, amplitude of the luminosity variation in the $B$ band and metallicity of the RR Lyrae stars. The reddening value we derive is $E(B-V)=0.15 \pm 0.04$ mag, assuming $[\mathrm{Fe} / \mathrm{H}]=-1.8$ dex for the metallicity. The $E(B-V)$ value and its r.m.s do not change if stars V2, V8, V9 and V27 are excluded. The $E(B-V)$ derived from the RR Lyrae stars is larger than the value of $E(B-V)=0.094 \pm 0.026 \mathrm{mag}$ derived by Schlegel et al. (1998), but still consistent in $1 \sigma$. The distance modulus of And XXI derived from the RR Lyrae stars using our reddening estimate is $(\mathrm{m}-\mathrm{M})_{0}=24.40 \pm 0.17$ mag. Our distance modulus is smaller than the one found by Conn et al. $\left[2012,(\mathrm{~m}-\mathrm{M})_{0}=24.59_{-0.07}^{+0.06} \mathrm{mag}\right]$ using the luminosity of the RGB tip, although compatible within $1 \sigma$. The difference between the two distance estimates is mostly due to differences in the adopted reddening values. Conn et al. (2012) use the reddening by Schlegel et al. (1998) which is $0.057 \mathrm{mag}$ smaller than our estimate from the RR Lyrae stars. If one adopts the same reddening value the two distance moduli become identical.

\section{CMD AND STELLAR POPULATIONS}

The CMD of And XXI obtained in the present study is shown in Figure 8, where in the left panel we plotted only sources located within the area delimited by the galaxy half-light radius and the ellipticity by Martin et al. (2009), whereas the right panel shows the CMD of all sources in the LBC field of view (FOV). To mitigate contamination from background galaxies and peculiar objects we selected our photometric catalog using the $\chi$ and Sharpness quality parameters provided by ALLFRAME and only retained sources with $-0.3 \leq$ Sharpness $\leq 0.3$ and $\chi<1.1$. The 


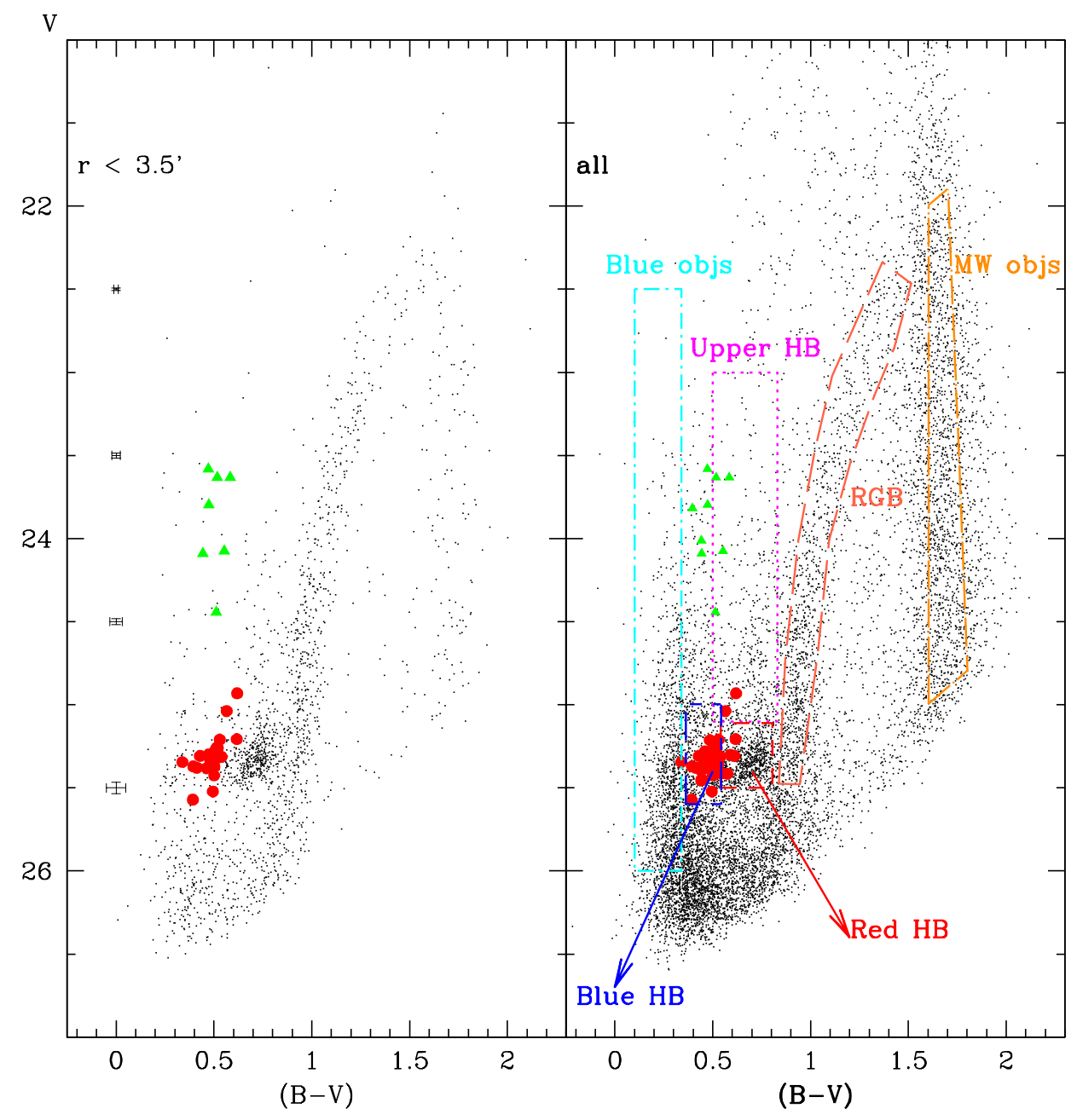

Fig. 8. - Left: CMD of the sources in our photometric catalog with $-0.3 \leq$ Sharpness $\leq 0.3, \chi<1.1$ and located within the area delimited by the galaxy half-light radius and the ellipticity by Martin et al. (2009). Red circles mark the RR Lyrae stars while the green triangles are ACs. Typical error bars are shown on the left. Right: As in the left panel, but considering sources in the whole LBC FOV. The regions in the CMD mark different selections performed to study the projected distributions (see Section 6). 
variable stars are plotted with red filled circles and green triangles for RR Lyrae stars and ACs, respectively. Prominent features of the CMD are the blue horizontal branch (HB) traced by the RR Lyrae stars, the red $\mathrm{HB}$ and the red giant branch (RGB). The sequence of stars at $B-V \sim 1.5-1.7 \mathrm{mag}$ and extending blueward around $V=22$ mag is mostly composed by foreground field stars. The relative occurrence of both RR Lyrae stars and ACs in And XXI suggests that the galaxy hosts different stellar generations. The CMD features and the position of the variable stars in the CMD were compared to the Padova isochrones obtained using the CMD 2.5 web interface (http : //stev.oapd.inaf.it/cgi-bin/cmd) which is based on models from Bressan et al. (2012). This is shown in Figure 9. Isochrones from 11 to 13 Gyr and $\mathrm{Z}=0.0003$ fit well the blue part of the RGB and the position of the RR Lyrae stars, (see top panels of Figure 9). Bressan et al. (2012) use for the Sun a value of $Z=0.0152$ hence the tracks at $\mathrm{Z}=0.0003$ correspond to $[\mathrm{Fe} / \mathrm{H}]=-1.7$ dex. The central and red part of the RGB together with red HB are well fitted by isochrones from 6 to 10 Gyr and enhanced metallicity ranging from $Z=0.0004$ to $Z=0.0006([\mathrm{Fe} / \mathrm{H}] \sim-1.5 \mathrm{dex})$, as shown in central and bottom panels of Figure 9 The intermediate age population seems to be dominant in And XXI given the best fit of the RGB with the 6-10 Gyr isochrones and the high number of HB-red stars (692) compared to HB-blue stars (368), and considering also that the contamination by background unresolved galaxies is much more prominent in the HB-blue. Another possible argument in favor of this hypothesis is the paucity of RRc stars. In the literature there are other systems with similar properties of metallicity and average period of the RRab stars which totally lack RRc stars, the Galactic globular clusters Ruprecht 106 (Rup 106, Kaluznv et al. 1995) and NGC 5824 (Meissner et al. 2006) that both are younger than other Galactic globular clusters with same metallicity.

The presence in And XXI of 9 ACs gives hints of a possible stellar population with age in the range of 1-2 Gyr. Indeed, Figure 10 shows that the position of the ACs in the CMD is well fitted by isochrones from 1 to 2 Gyr with metallicity in the range $\mathrm{Z}=0.0001-0.0006$. Furthermore, above the $\mathrm{HB}$ we observe a sequence of objects that likely belong to this young stellar component. We should remind that the origin of ACs is still a matter of debate in the literature, with the two most accepted scenarios being that they either represent young single stars produced by a relatively recent episode of star formation, or that they formed from mass transfer in binary systems as old as the other stars in the galaxy. Both channels may produce ACs, however, perhaps a distinction can be made between the few ACs found in old stellar systems like globular clusters and ultra faint dwarf galaxies (UFDs) like Hercules (Musella et al. 2012), that likely originate from binaries, and the larger numbers of ACs found in galaxies with gas and recent star formation like Leo T (Clementini et al. 2012) among the UFDs and And XIX (Paper I) and And XXI among the M31 satellites, that likely are bona fide young stars.

\section{PROJECTED DISTRIBUTIONS}

To check whether the objects above the HB are young stars belonging to And XXI and not contaminant sources, we selected stars from the CMD of all the sources in the LBC FOV which have color in the range $0.50<(B-V)<0.83 \mathrm{mag}$ and magnitude $23.0<V<25.1 \mathrm{mag}$ (see right panel of Figure 8 for the different selections). We plotted the selected objects in a RA and DEC map (see bottom left panel of Figure 11) and found evidence of a clustering about $\sim 1$ arcmin $(0.017 \mathrm{deg})$ southwest from And XXI center. Although slightly offset with respect to the center of And XXI these young objects correlate with the general projected distribution of the CMD-selected RGB and redHB stars, as shown in Figure 11, and likely are galaxy members. The distribution of RGB stars (middle-left panel of Figure 11) is consistent with And XXI position angle and ellipticity as given by Martin et al. (2009), while the red-HB stars (middle-right panel of Figure 11) appear to be more concentrated toward the center than the RGB stars. That older stars have a more extended distribution compared to younger populations is a well known, general feature seen in dwarf galaxies (see e.g. Held et al. 2001; Tolstov et al. 2004; Clementini et al. 2005; Bellazzini et al. 2014).

The blue objects between $0.1<(B-V)<0.4$ mag and $22.5<V<26$ mag are most proba- 


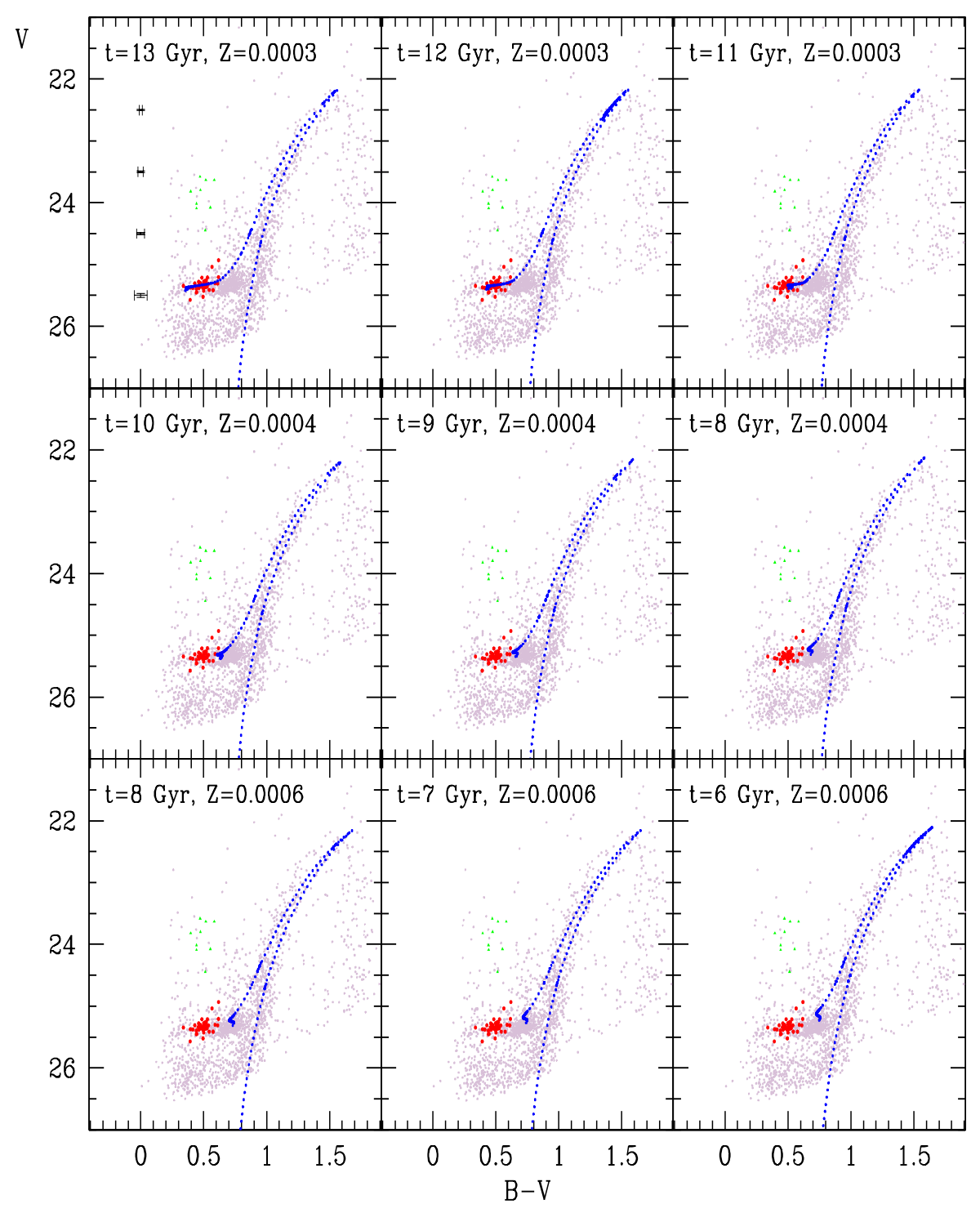

Fig. 9.- CMD of And XXI overlaid by Padova stellar isochrones (Bressan et al. 2012) with different age (13-6 Gyr) and metallicity ( $\mathrm{Z}=0.0003, \mathrm{Z}=0.0004$ and $\mathrm{Z}=0.0006$; from top to bottom). Red filled circles are RR Lyrae stars, the green filled triangles are ACs. 


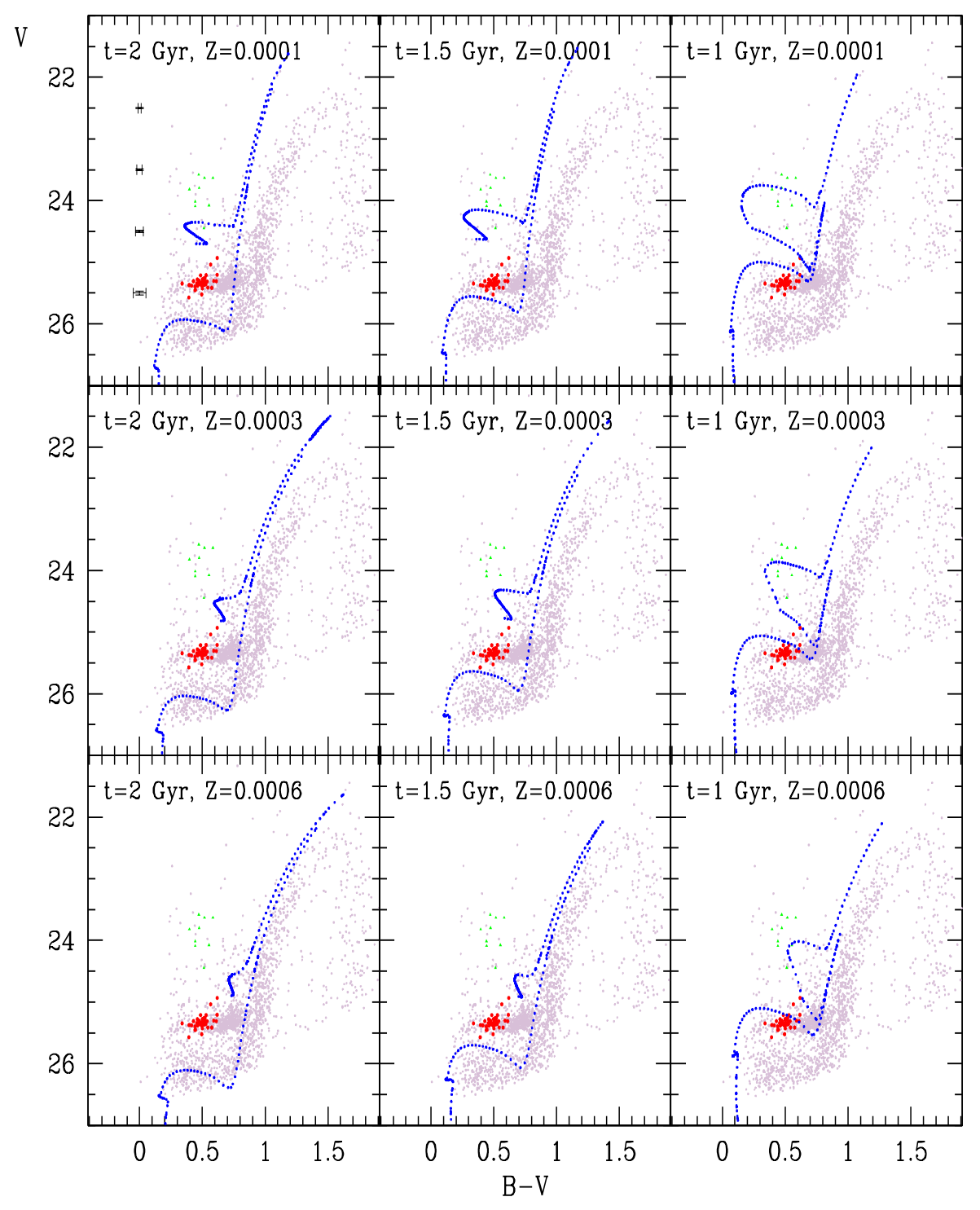

Fig. 10. - Same as in Figure 9, but for isochrones of 1, 1.5 and 2 Gyr and metallicity $Z=0.0001, Z=0.0003$ and $\mathrm{Z}=0.0006$ (from top to bottom). 


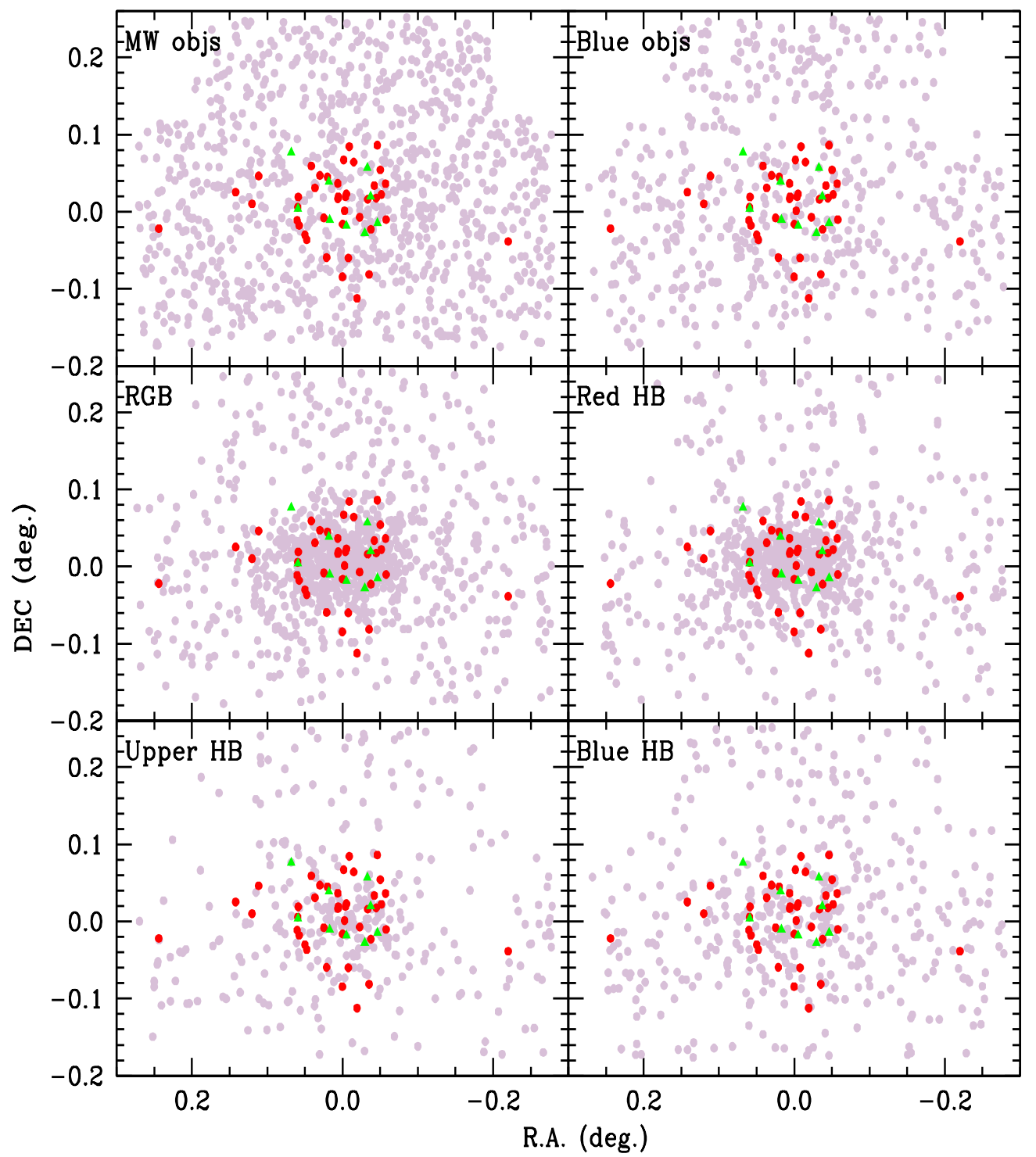

Fig. 11. - Projected distribution of selected samples of objects in the CMD of the total LBC FOV. Superimposed are the RR Lyrae stars (red, filled circles) and the ACs (green, filled triangles). 
bly unresolved galaxies, as discussed in Paper I. Their projected distribution (top right panel in Figure 11) is homogeneous over the whole LBC FOV, hence confirming that they are contaminant sources. The red objects between $1.5<(B-V)<$ $2.1 \mathrm{mag}$ and $26<V<21 \mathrm{mag}$ are MW foreground stars and are equally distributed all over the FOV (top left panel in Figure 11). Since there is a strong contamination from unresolved galaxies in the blue part of the CMD, it is a hard task to separate out the blue-HB members of And XXI. We selected as blue-HB members the stars falling in the CMD region $0.35<(B-V)<0.65 \mathrm{mag}$ and $25.2<V<25.7 \mathrm{mag}$, as defined by the RR Lyrae variables identified in this work. In the projected distribution, the blue HB stars (bottom right panel in Figure 11) are spread almost homogeneously over the galaxy and although the contamination from background sources may be significant, the HB-selected stars follow the galaxy position angle.

In order to identify particular structures in And XXI, we built isodensity maps of the RGB, red-HB and upper-HB stars. They are shown in Figure 12. Stars were binned in $1.2^{\prime} \times 1.2^{\prime}$ boxes and smoothed by a Gaussian kernel of full width at half maximum (FWHM) of $1.2^{\prime}$ (0.02 deg). The first contour levels are $3 \sigma$ above the sky background. Directions to M31 and And XIX, which is the biggest satellite in the neighbourhood of And XXI, are also shown in the figure. The projected relative distance of And XXI to M31 and And XIX is of $\sim 130 \mathrm{kpc}$ and $\sim 100 \mathrm{kpc}$, respectively. The RGB stars are spread over the LBC FOV for more than twice the galaxy $\mathrm{r}_{h}$. This was already clear for the presence of RR Lyrae stars far from the galaxy center. Up to $6-7$ arcmin $(\sim 0.11$ deg) from the center the isodensity contours well follow the position angle given by Martin et al. (2009). Right after 7 arcmin the isodensities appear to be twisted and in the east part of the LBC FOV are distorted in the direction of And XIX. A sort of arm highlighted by the presence of one RR Lyrae star extends in the direction of M31. The contours of red-HB and RGB stars have a similar distribution, while the upper-HB stars have different center and orientation when compared to the other two populations. The three populations show overdensities in the east, north and west directions. Two RR Lyrae stars are located at the position of the east and west overdensities, thus endorsing the presence of sub-structures far from And XXI center.

Figure 13 shows the cumulative radial distributions of selected samples of stars located inside twice the $\mathrm{r}_{h}$ of And XXI. We performed a twosample Kolmogorov-Smirnov (K-S) test between these radial distributions. The RGB (green line) and red-HB star (blue line) distributions shown in Figure 13 are indistinguishable and likely arise from the same stellar generation. In fact, the K-S test provides a $\mathrm{p}$-value as large as $\mathrm{p}=0.84$, whereas the K-S test between upper-HB population (red line) and RGB stars gives a $\mathrm{p}$-value of only $\mathrm{p}=0.24$.

Finally, though the number of variables (RRLs+ ACs) is very small when compared to the number RGB stars the K-S test gives $\mathrm{p}=0.49$ between the two samples.

\section{MERGING?}

In Section 3.1 we showed the presence of two peaks in the period distribution of And XXI RRab stars. The double peak in the period distribution can be a clue for the presence of two separate populations of RR Lyrae stars, with slightly different age and/or metallicity. Selecting stars with period within $1 \sigma$ from the two peaks in period, we find an average magnitude of $V=25.35 \pm 0.08 \mathrm{mag}$ for stars with $\mathrm{P}_{1}=0.60(\sigma=0.02) \mathrm{d}$ and $V=25.25 \pm 0.10$ mag for stars with $\mathrm{P}_{2}=0.68(\sigma=0.03) \mathrm{d}$. Although the $0.1 \mathrm{mag}$ difference between $\mathrm{P}_{1}$ and $\mathrm{P}_{2}$ RR Lyrae stars is within the range of errors, it goes in the direction that the longer period $R R$ Lyrae stars $\left(\mathrm{P}_{2}\right)$ are brighter than the shorted period $\left(\mathrm{P}_{1}\right)$ ones, possibly due to a lower metal content. A difference in metallicity of 0.4 dex can explain such magnitude difference according to Clementini et al. (2003) and Gratton et al. (2004) slope of the luminosity-metallicity relation of RR Lyrae stars. Since the RR Lyrae stars are produced by a population older than $10 \mathrm{Gyr}$, the double-peaked period distribution might suggest that either the primordial environment of And XXI was enriched in only 2 Gyr or that the galaxy RR Lyrae stars arise from two different small galaxies that merged to form And XXI.

Furthermore, the RGB of And XXI seems to be bifurcated, giving a further hint for the presence of two slightly different old populations. To show 

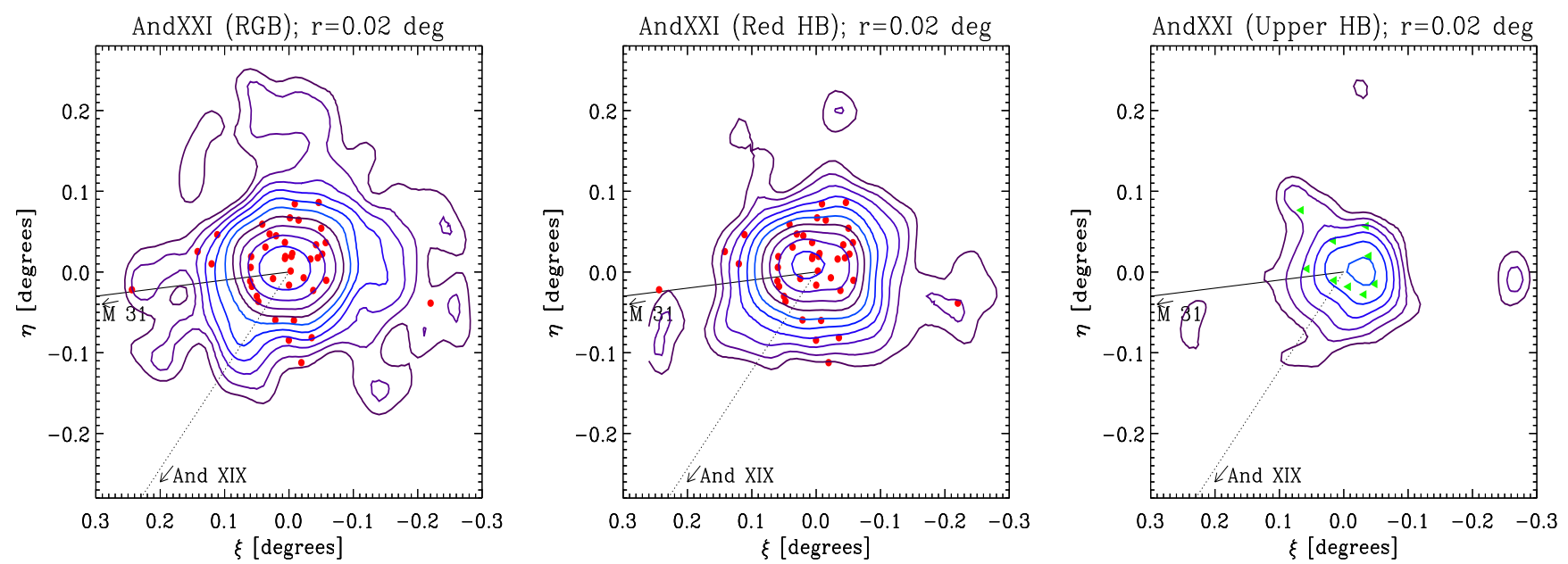

Fig. 12.- Isodensity contours of three selected samples of stars in And XXI. $\xi$ and $\eta$ are the RA and DEC computed from the center of the galaxy given by Martin et al. (2009) and $r$ is the FWHM of the Gaussian kernel. Left panel: RGB stars, Center panel: red-HB stars, Right panel: upper-HB stars. RR Lyrae stars and ACs are also plotted as red filled circles and green filled triangles, respectively.

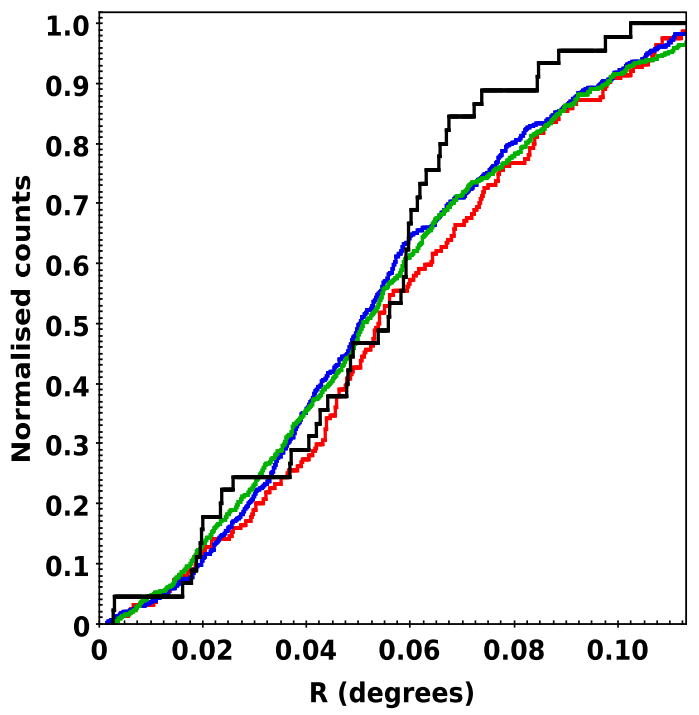

Fig. 13.- Cumulative radial distributions of selected samples of sources in the CMD of And XXI. Green line: cumulative distribution for the RGB stars, blue line: distribution of the red-HB stars, red line: distribution of the upper-HB sources, black line: distribution of the variable stars. that the spread of And XXI RGB is real and not due to contamination or photometric errors, we matched our catalog with the list of spectroscopic members of And XXI identified by Collins et al. (2013). The latter are marked by blue open circles in the CMD of Figure 14. They are mostly RGB stars that exhibit a wide spread in color, at a given luminosity, due to age and/or metallicity differences. As shown in the left panel of Figure14 the member stars are enclosed within the $12 \mathrm{Gyr}$, $\mathrm{Z}=0.0002$ and the $13 \mathrm{Gyr}, \mathrm{Z}=0.0004$ tracks. In addition the red-HB of And XXI also separates in two clumps with a difference of $\sim 0.15$ in $V$ magnitude (see left panel of Figure 15). A further argument in support of the merging scenario is the different projected distributions of the selected samples of stars in And XXI.

As early suggested by Coleman et al. (2004) and later supported by Yozin \& Bekki (2012) with numerical simulations, the MW dSph galaxy Fornax resulted from merging of two dwarf galaxies. The visible remnant of this collision was detected by Coleman et al. (2004) as a small overdensity of young stars forming a shell structure displaced from Fornax center. The young stars in And XXI (see Section 5) are indeed located in an aggregation displaced from the center of the galaxy, which resembles the overdensity found in Fornax 


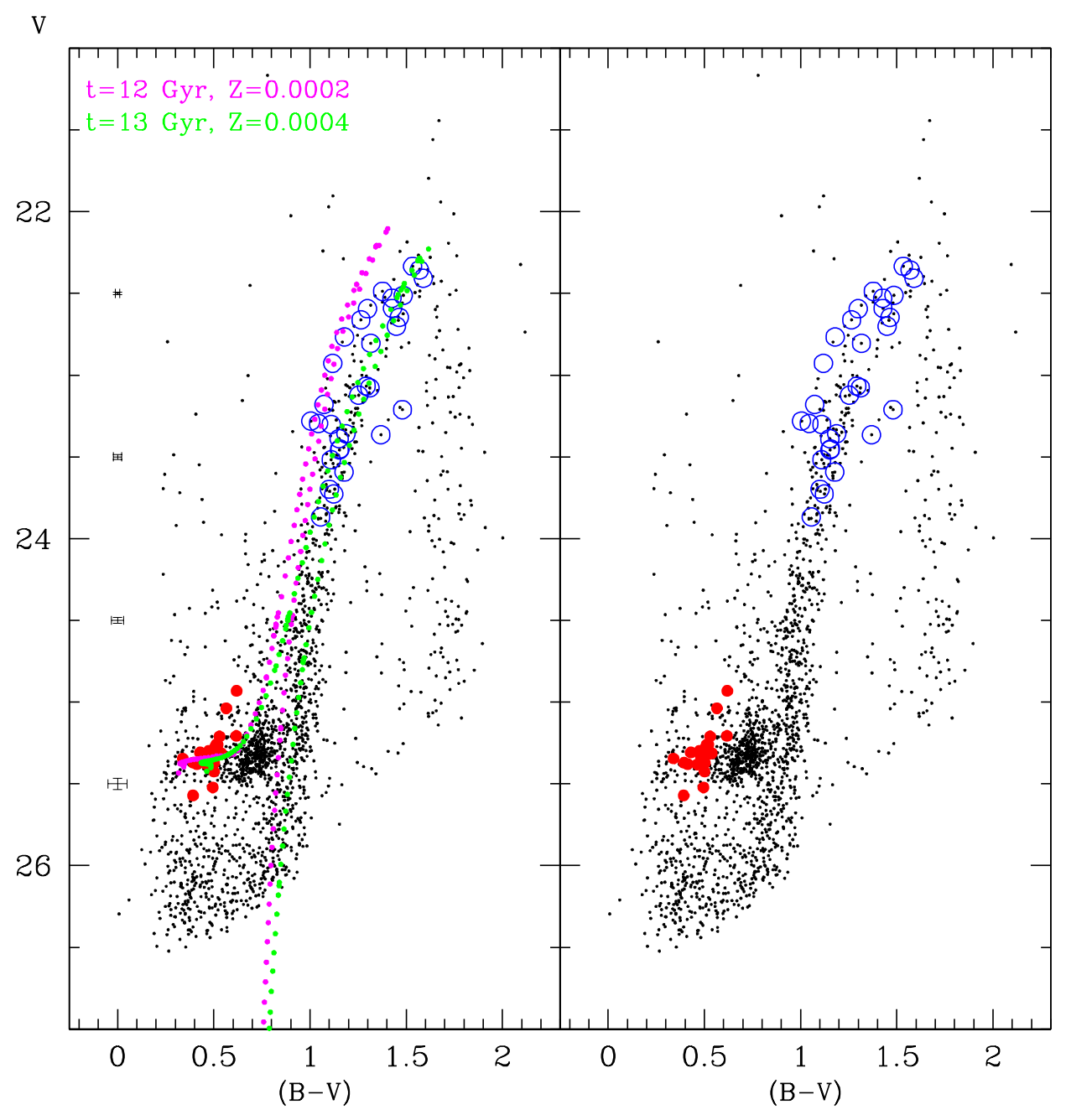

Fig. 14. - CMD of And XXI with marked as open blue circles stars with membership spectroscopically confirmed by Collins et al.(2013). Dashed lines are the Bressan et al.'s isochrones for 12 Gyr, Z=0.0002 (magenta line) and $13 \mathrm{Gyr}, \mathrm{Z}=0.0004$ (green line), respectively. Red filled circles are RR Lyrae stars. Typical photometric error bars are shown on the left. 
by Coleman et al. (2004). Furthermore, And XXI variable stars seem to be placed in separate shells around the galaxy center (see Figure 4). These features suggest also for And XXI a merging scenario.

According to Amorisco et al. (2014), among the M31 satellites, And II is another example of dwarf merging remnant. This would explain the stellar stream they detected kinematically in the galaxy. The presence of two different old populations in And II is supported also by the analysis of the CMD and by the properties of the variable stars. The RR Lyrae stars of And II were studied by Pritzl et al. (2004). Using the periods in Table 2 of Pritzl et al. (2004), we performed a multi-gaussian fit and found two separate peaks, a first one at $\mathrm{P}=0.52 \mathrm{~d}$ with $\sigma=0.05 \mathrm{~d}$ and a second one at $\mathrm{P}=0.68 \mathrm{~d}$ with $\sigma=0.01 \mathrm{~d}$. Hence, similarly to And XXI, also And II seems to host two distinct old stellar populations with different metallicity/age and corresponding different RR Lyrae populations, likely belonged to the two galaxies that merged in the past. The left panel of Figure 16 shows the CMD of And II we obtained using HST archive data (Prop ID 6514, PI: Gary Da Costa). The galaxy RGB clearly bifurcates in two arms and blue and red HBs also seem to split. CMD and variable stars in And II show properties very similar to And XXI (central panel of Fig. 16). For comparison the right panel of Fig. 16 shows the
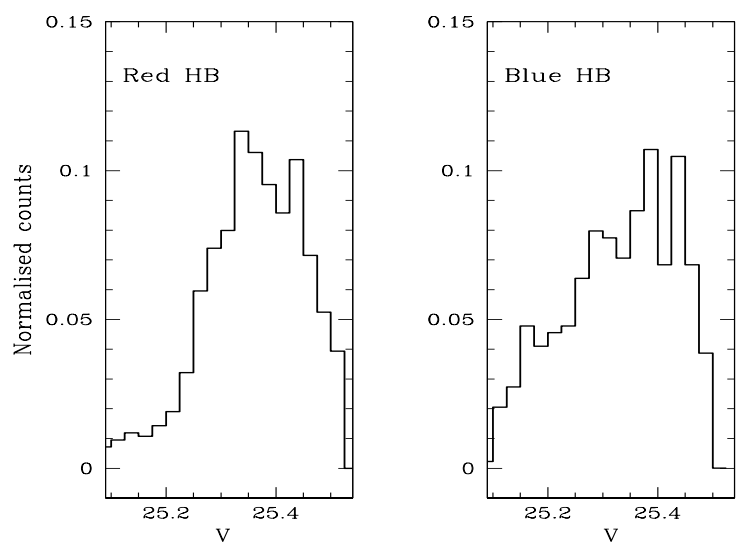

Fig. 15.- Histogram of the $V$ magnitudes for the stars in the Red (left panel) and Blue (right panel) HB. de-reddened CMD of And XIX (Paper I) inside the galaxy $\mathrm{r}_{h}$ of $6.2 \operatorname{arcmin}$. The $\mathrm{r}_{h}$ of And XIX is approximately twice that of And XXI and the contamination by foreground and background objects is much higher. Still the RGB of And XIX is much narrower than the RGB of And XXI and And II. In conclusion, we speculatively attribute some of the features of the CMD and RR Lyrae population of And XXI to the presence of two slightly different stellar populations belonging to two dwarf galaxies that merged in the past.

Recently, Deason et al. (2014) investigated the frequency of merging between dwarf galaxies in the LG using the ELVIS simulations. They found that the frequency of satellite-satellite merging in the host virial radius of the MW and M31 is of the order of $10 \%$ and that this frequency doubles for satellites outside the virial radius. Considering the large number of satellites surrounding the MW and M31 these predictions are consistent with both And II and And XXI being the result of satellite merging.

\section{CONCLUSIONS}

We have discovered a total of 50 variable stars in And XXI, of which 41 are RR Lyrae stars and 9 are ACs. From the average period of the RRab stars $\left\langle\mathrm{P}_{\mathrm{ab}}\right\rangle=0.64 \mathrm{~d}$ and the Bailey diagram we classify And XXI as an Oo-II/Int object. Using the variable stars and the CMD we found evidence for the presence in And XXI of two major stellar populations, a first one 12 Gyr old with $[\mathrm{Fe} / \mathrm{H}]=-$ 1.7 dex and a second, more conspicuous one with an age of $6-10 \mathrm{Gyr}$ and $[\mathrm{Fe} / \mathrm{H}]=-1.5$ dex. Furthermore, the discovery of $9 \mathrm{ACs}$ traces also the presence in And XXI of a stellar population as young as 1-2 Gyr. This is similar to what we found in And XIX (Paper I), but in And XXI the 6-10 Gyr stellar component is much more dominant. Other dwarf satellites of M31 contain RR Lyrae stars as well as prominent red clumps in the CMD (e.g. see the CMD of And I and And III in Fig.1 by Pritzl et al. 2005). Mancone \& Sarajedini (2008) found that the population of And V is mostly composed of stars 8-10 Gyr old. Our results on the stellar population of And XXI and And XIX together with the above further literature evidences seem to suggest that a global event triggered star formation in the dwarf galaxy satellites of M31 


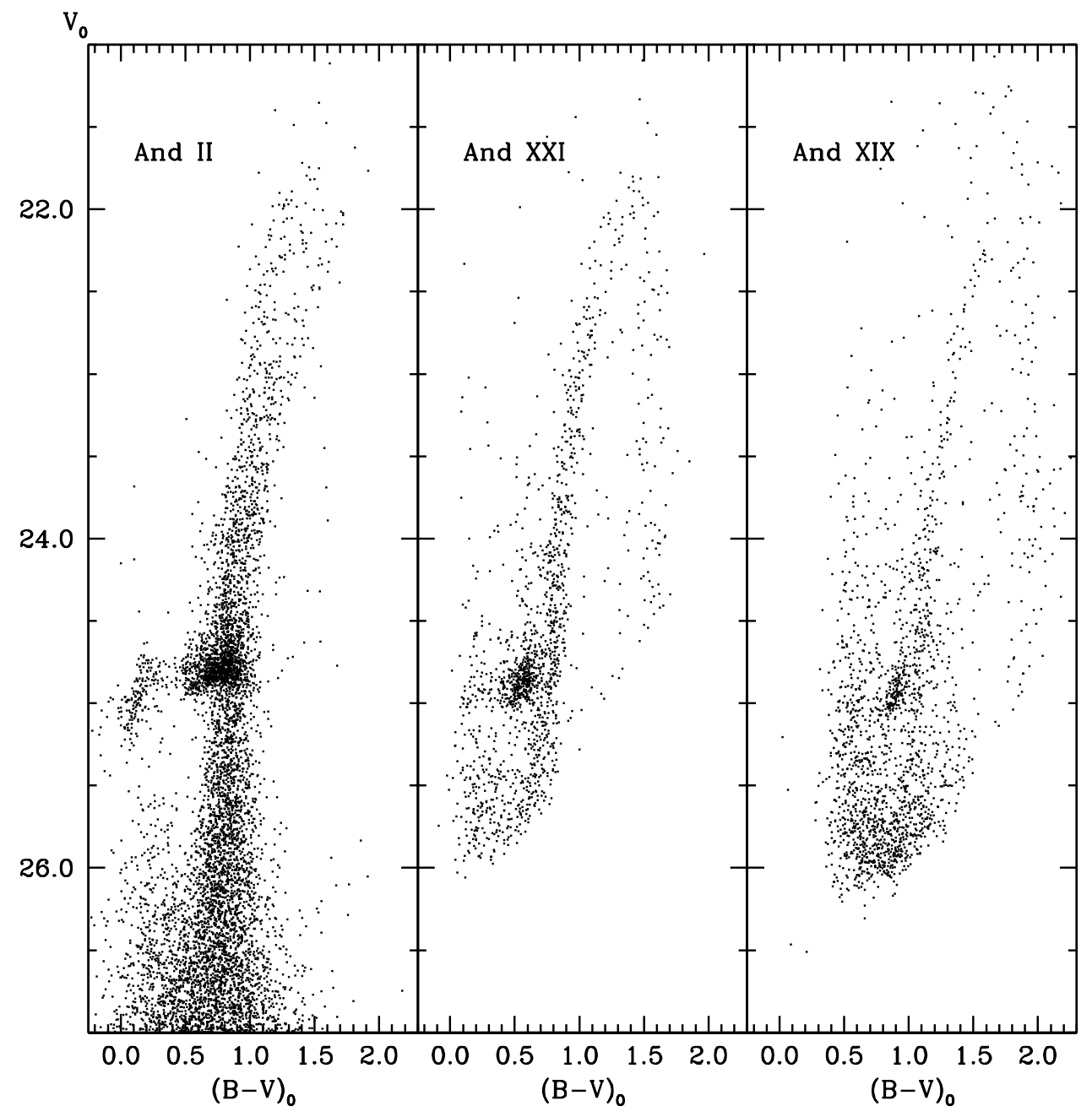

Fig. 16.- Comparison of the de-reddened CMD of And II (left panel) we obtained from HST archive data, with the CMDs of And XXI (central panel, this work) and And XIX (Paper I). Only stars within the respective half-light radii are shown for And XXI and And XIX. 
about 6-10 Gyr ago. An engine triggering this common star formation episode could possibly be the fly-by encounter of the MW and M31 reported by Zhao et al. (2013)

The projected distribution of sources properly selected from the CMD of And XXI shows the existence of an overdensity of young/intermediate age stars in a region slightly off from the galaxy center. We discussed this evidence in light of And XXI possibly being the result of a merging between two dwarf galaxies. This hypothesis is corroborated by the possible presence in the galaxy of two different RR Lyrae populations as well as peculiar features in the CMD (wide RGB, bifurcated red HB) and from the presence of shell-like structures. If future spectroscopic observations will confirm And XXI to be a merged galaxy, it would become the second such a satellite in M31, after And II.

We warmly thank P. Montegriffo for the development and maintenance of the GRATIS software, G. Battaglia for providing the software to compute And XXI's density maps and A. Veropalumbo for software assistance. Financial support for this research was provided by PRIN INAF 2010 (PI: G. Clementini) and by Premiale LBT 2013. The LBT is an international collaboration among institutions in the United States, Italy, and Germany. LBT Corporation partners are The University of Arizona on behalf of the Arizona university system; Istituto Nazionale di Astrofisica, Italy; LBT Beteiligungsgesellschaft, Germany, representing the Max-Planck Society, the Astrophysical Institute Potsdam, and Heidelberg University; The Ohio State University; and The Research Corporation, on behalf of The University of Notre Dame, University of Minnesota, and University of Virginia. We acknowledge the support from the LBT-Italian Coordination Facility for the execution of observations, data distribution, and reduction. Facility: LBT

\section{REFERENCES}

Amorisco, N. C., Evans, N. W., van de Ven, G 2014, Natur, 507, 335

Bailey, S. I. 1902, Annals of Harvard College Observatory, 38,1

Bellazzini, M., Beccari, G., Fraternali, F., et al. 2014, A\&A, 566A, 44
Bressan, A., Marigo, P., Girardi, et al. 2012, MNRAS, 427, 127

Cacciari, C., Corwin, T. M., \& Carney, B. W. 2005, AJ, 129, 267

Clementini, G., Contreras Ramos, R., Federici, L., et al. 2011, ApJ, 743, 19

Clementini, G., Cignoni, M., Contreras, R., et al. 2012, ApJ, 756, 108

Clementini, G., Di Tomaso, S., Di Fabrizio, L., et al. 2000, AJ, 120, 2054

Clementini, G., Gratton, R., Bragaglia, et al. 2003, AJ, 125,1309

Clementini, G., Ripepi, V., Bragaglia, A., et al. 2005, MNRAS, 365, 734

Coleman, M., Da Costa, G. S., Bland-Hawthorn, J., et al. 2004, AJ, 127, 832

Collins, M. L. M., Chapman, S. C., Rich, R. M., et al. 2013, ApJ768, 172

Conn, A. R., Ibata, R. A., Lewis, G. F., et al. 2012, ApJ, 758, 11

Cusano, F., Clementini, G., Garofalo, A., et al. 2013, ApJ, 779, 7

Deason, A., Wetzel, A., Garrison-Kimmel, S. 2014, ApJ, 794, 115

Gratton, R. G., Bragaglia, A., Clementini, G., et al. 2004, A\&A, 421, 937

Hammer, F., Yang, Y., Fouquet, S., et al. 2013, MNRAS, 431, 3543

Held, E. V., Saviane, I., Momany, Y., Rizzi, L., \& Bertelli, G. 2001, ApSSS, 277, 331

Ibata, R. A., Lewis, G. F., Conn, A. R., et al. 2013, Natur, 493, 62

Kaluzny, J., Krzeminski, W., Mazur, B. 1995, AJ, 110, 2206

Madore B. F. 1982, ApJ, 253, 575

Mancone, C., \& Sarajedini A. 2008, AJ, 136, 1913

Marconi, M., Fiorentino, G., \& Caputo, F. 2004, A\&A, 417, 1101 
Martin, N. F., McConnachie, A. W., Irwin, et al. 2009, ApJ, 705, 758

Martin, N. F., Ibata, R. A., McConnachie, A. W., et al. 2013, ApJ, 776, 80

McGaugh, S., \& Milgrom, M. 2013, ApJ, 775, 139

Meissner, F. \& Weiss, A 2006, A\&A, 456, 1085

Milgrom, M. 1983, ApJ, 270, 365

Musella, I., Ripepi, V., Marconi, M., et al. 2012, ApJ, 756, 121

Oosterhoff, P. T. 1939, The Observatory, 62, 104

Pawlowski, M. S., Famaey, B., Jerjen, H., et al. 2014, MNRAS, 442, 2362

Piersimoni, A. M., Bono, G., \& Ripepi, V. 2002, AJ, 124, 1528

Pritzl, B. J., Jacoby, G. H., \& Da Costa, G. S. 2004, AJ, 127, 318

Pritzl, B. J., Armandroff, T. E., Jacoby, G. H., \& Da Costa, G. S. 2005, AJ, 129, 2232

Ripepi, V., Marconi, M., Moretti, M. I., et al. 2014, MNRAS, 437, 2307

Schlegel, D. J., Finkbeiner, D. P., \& Davis, M. 1998, ApJ, 500, 525

Soszynski, I., Poleski, R., Udalski, A., et al. 2008, AcA, 58, 163

Stetson, P. B. 1987, PASP, 99, 191

Stetson, P. B. 1994, PASP, 106, 250

Tolstoy, E., Irwin, M. J., Helmi, A.,et al. 2004, ApJ, 617, L119

van den Bergh, S. 1975, StarsandStellarSystems, 9, 509, ed. A. R. Sandage, M. Sandage, and J. Kristian (Chicago: University of Chicago Press)

Yozin, C., \& Bekki, K. 2012, ApJ, 756L, 18

Zhao, H., Famaey, B., Lüghausen, F., \& Kroupa, P. 2013, A\&A, 557L, 3

Zolotov, A., Willman, B., Brooks, A. M., et al. 2009, ApJ, 702, 1058

This 2-column preprint was prepared with the AAS LATEX macros v5.2. 